\title{
A systematic review of proxy-report questionnaires assessing physical activity, sedentary behavior and/or sleep in young children (aged 0-5 years)
}

Jelle Arts $^{1 *}$ (D, Jessica S. Gubbels², Arnoud P. Verhoeff ${ }^{3,4}$, Mai. J. M. Chinapaw ${ }^{1}$, Annelinde Lettink ${ }^{1}$ and Teatske M. Altenburg ${ }^{1}$

\begin{abstract}
Background: Accurate proxy-report questionnaires, adapted to the child's developmental stage, are required to monitor 24-h movement behaviors in young children, especially for large samples and low-resource settings.

Objectives: This review aimed to summarize available studies evaluating measurement properties of proxy-report questionnaires assessing physical activity, sedentary behavior and/or sleep in children aged 0-5 years.

Methods: Systematic literature searches were carried out in the PubMed, Embase and SPORTDiscus databases, up to January 2021. For physical activity and sedentary behavior questionnaires this is a review update, whereas for sleep questionnaires we included all relevant studies published up to now. Studies had to evaluate at least one of the measurement properties of a proxy-report questionnaire assessing at least duration and/or frequency of physical activity, sedentary behavior and/or sleep in 0- to 5-year-old children. The COnsensus-based Standards for the selection of health Measurement INstruments (COSMIN) guideline was used to evaluate the quality of evidence.
\end{abstract}

Results: Thirty-three studies were included, examining a total of 37 questionnaires. Ten questionnaires were designed for infants, two for toddlers, 11 for preschoolers, and 14 for a broader age range targeting multiple of these age groups. Twenty questionnaires assessed constructs of sleep, four assessed constructs of physical activity, two assessed screen behavior, five assessed constructs of both physical activity and sedentary behavior, and six assessed constructs of all 24-h movement behaviors. Content validity was evaluated for six questionnaires, structural validity for two, internal consistency for three, test-retest reliability for 16, measurement error for one, criterion validity for one, and construct validity for 26 questionnaires. None of the questionnaires were considered sufficiently valid and/or reliable for assessing one or more movement behaviors in 0 - to 5 -year-old children, and the quality of evidence was mostly low or very low.

Conclusions: Valid and/or reliable questionnaires assessing 24-h movement behaviors in 0- to 5-year-olds are lacking. High-quality studies are therefore required, to develop proxy-report questionnaires and evaluate their measurement properties.

\footnotetext{
*Correspondence: j.arts@amsterdamumc.nl

${ }^{1}$ Department of Public and Occupational Health, Amsterdam UMC, Vrije Universiteit Amsterdam, Amsterdam Public Health research institute, Van der Boechorststraat 7, 1081, BT, Amsterdam, The Netherlands

Full list of author information is available at the end of the article
} permits use, sharing, adaptation, distribution and reproduction in any medium or format, as long as you give appropriate credit to the original author(s) and the source, provide a link to the Creative Commons licence, and indicate if changes were made. The images or other third party material in this article are included in the article's Creative Commons licence, unless indicated otherwise in a credit line to the material. If material is not included in the article's Creative Commons licence and your intended use is not permitted by statutory regulation or exceeds the permitted use, you will need to obtain permission directly from the copyright holder. To view a copy of this licence, visit http://creativecommons.org/licenses/by/4.0/. The Creative Commons Public Domain Dedication waiver (http://creativeco mmons.org/publicdomain/zero/1.0/) applies to the data made available in this article, unless otherwise stated in a credit line to the data. 
PROSPERO registration number: CRD42020169268.

Keywords: 24-h movement behaviors, Infants, Toddlers, Preschoolers, Questionnaires, Parent-report, Measurement properties, Validity, Reliability

\section{Background}

Establishing healthy movement behaviors in early childhood is necessary to support the growth and development of young children and the maintenance of their long-term health [1-4]. Recent studies indicate the importance of the combination of all 24-h movement behaviors, encompassing physical activity, sedentary behavior, and sleep [5-10]. Valid, reliable, responsive, affordable, and feasible measurement instruments adapted to the child's developmental stage are required to monitor 24-h movement behaviors in young children. Direct observation is considered a suitable criterion measure of different movement behaviors in children $[11,12]$. However, observation can be very time consuming and thus costly, and is thereby not feasible to use on a large scale. Alternatively, physical activity, sedentary behavior and sleep in young children can be measured using accelerometers $[3,12,13]$. Although accelerometers are considered valid and reliable for measuring movement behaviors in children and adolescents $[14,15]$, its validity is yet to be obtained for the youngest age group (i.e., infants: $0-1$ year old) [13]. In addition, accelerometer output is processed by a number of subjective decisions in order to translate acceleration data into time estimates of movement behaviors. Specifically, it is unknown which analyses methods provide the most accurate classification of physical activity, sedentary behavior and sleep in infants. Additionally, current procedures do not take into account that accelerometer output in very young children may reflect movements of others, e.g., that of parents who carry their child or push them in a stroller [16]. Alternative to observation and accelerometry, parent- or caregiver (proxy-) report questionnaires can be used as a measurement instrument for children's 24-h movement behaviors. Questionnaires can be used to assess movement behaviors on a large scale in a relatively convenient and affordable way, with the additional advantage of obtaining information about the type (e.g., active play, screen time) and context (e.g., outside, alone) of the behavior. Unfortunately, proxy-report questionnaires have their own limitations such as recall and social desirability bias [17]. Furthermore, the intermittent and unstructured pattern of movement behaviors in young children complicates accurate reporting of these behaviors [17].

To date, a number of proxy-report questionnaires have been developed to assess physical activity, sedentary behavior and/or sleep in 0 - to 5-year-old children.
A few systematic reviews examined the measurement properties of these questionnaires in young children [18-21], searching the literature up to 2014 for questionnaires on sleep [18], up to 2015 for sedentary behavior [20] and up to 2018 for physical activity [19, 21]. However, concerning questionnaires on sleep, questionnaires for the youngest age group were not evaluated (i.e., 0 - to 2 -year-old children) [18]. Unfortunately, these reviews did not identify any questionnaires that can be considered both reliable and valid for assessing physical activity, sedentary behavior and/ or sleep in children aged $<5$ years [18-21]. Although a review published in 2011 [22] and updated in 2020 [23] provided an overview of the psychometric analyses performed at the available pediatric sleep questionnaires, including questionnaires for 0 - to 5 -year-old children, measurement property results were not reported in the review update [23]. In addition, quality of evidence was not considered in both reviews, limiting the conclusions that can be drawn regarding the best available questionnaires $[22,23]$. Similarly, a recently published systematic review provided an overview of measurement tools used to assess screen time in 0- to 6-yearold children [22]. Although measurement properties of the available questionnaires were reported, a comprehensive evaluation of the measurement properties, including quality of evidence, was lacking [24]. Furthermore, as the research interest of all 24-h movement behaviors in young children has grown, new questionnaires might have been developed over the last few years. For this reason, an update of previous reviews is required, including physical activity, sedentary behavior and sleep. To be able to select the most appropriate questionnaires to assess 24-h movement behaviors, an overview of the characteristics and measurement properties of the available questionnaires for young children is highly warranted.

Therefore, the purpose of this review was to summarize all studies examining measurement properties (e.g., reliability and validity) of proxy-report questionnaires assessing physical activity, sedentary behavior and/or sleep in children aged $0-5$ years. We evaluated the quality of evidence for each measurement property, including a methodological quality assessment of included studies. Additionally, we provided an overview of the characteristics of the evaluated questionnaires (e.g., the target population and format of the questionnaires). 


\section{Methods}

We registered this review on PROSPERO (international prospective register of systematic reviews; registration number: CRD42020169268) and followed the Preferred Reporting Items for Systematic Reviews and Meta-Analyses (PRISMA) guidelines [25].

\section{Literature search}

Systematic literature searches were carried out in PubMed, Embase, and SPORTDiscus. For physical activity and sedentary behavior questionnaires this review is an update of previous reviews [19-21], whereas for sleep questionnaires we included all relevant studies published up to now. Therefore, separate searches were carried out, i.e., one for physical activity and sedentary behavior, and one for sleep. The full search strategies can be found in Additional file 1.

For physical activity and sedentary behavior questionnaires, we searched the literature from December 2015 (i.e., last search period for sedentary behavior questionnaires [20]) until December 2019. In PubMed more overlap in time was maintained (search from May 2015), as our previous searches showed that the PubMed time filter can be inaccurate due to incorrect labeling of publication dates. For sleep questionnaires there was no lower limit for publication date, and databases were searched up until January 2020. A combined update search (i.e., physical activity, sedentary behavior and sleep) was completed on 6 January 2021.

Both search strategies focused on terms related to young children (e.g., infant, toddler, preschooler), proxyreport measures (e.g., questionnaire, proxy-report) and measurement properties (e.g., reliability, reproducibility, validity). For the physical activity and sedentary behavior search, these terms were used in AND-combination with terms related to physical activity (e.g., motor activity, exercise) OR sedentary behavior (e.g., stationary behavior, screen-time). For the sleep search, these terms were used in AND-combination with terms related to sleep (e.g., bedtime, nap). In all databases, studies in animals and children with a variety of diseases or disorders (e.g., autism, attention deficit disorder) were excluded using NOT-combinations.

\section{Inclusion and exclusion criteria}

Studies were included when: (1) the study evaluated at least one of the measurement properties of a proxyreport questionnaire assessing physical activity, sedentary behavior and/or sleep; (2) the proxy-report questionnaire under study reported at least data on the duration and/or frequency of physical activity, sedentary behavior and/or sleep; (3) the study included apparently healthy children, born term $(>37$ weeks), aged $<5$ years or a wider age range with the results for 0- to 5-year-old children described separately; (4) the study was published in English in a peer-reviewed journal; and (5) the full-text was available.

Studies were excluded when (1) measurement properties were evaluated in a specific subpopulation or clinical sample (e.g., children with sleep problems); (2) construct validity was only evaluated by examining the relationship between the questionnaire and a non-similar construct (e.g., between physical activity and body mass index (BMI)); (3) structural validity and/or internal consistency were evaluated for questionnaires that represent a formative measurement model as these analysis are not relevant when the items of a scale or subscale form or cause the construct and are therefore not supposed to be correlated (e.g., items assessing different physical activities that together form the construct duration of total physical activity) [26, 27]; (4) responsiveness was evaluated without using a comparison measure to assess a questionnaire's ability to detect change (e.g., accelerometer).

\section{Selection procedures}

Articles were imported in EndNote X9.1, and subsequently duplicate articles were removed. Titles and abstracts of potentially relevant articles were scanned by two independent researchers (JA and TA) using Rayyan. Next, full texts were obtained and independently screened for eligibility by the same two researchers. Additionally, reference lists of all full-text articles were screened for additional studies. Disagreements were resolved through discussion.

\section{Inclusion of studies from previous reviews}

To draw definite conclusions regarding the best available questionnaires, we also included studies from the three aforementioned reviews evaluating questionnaires assessing physical activity and sedentary behavior [1921]. As these reviews were not restricted to questionnaires aimed to assess young children's behaviors, only the studies that evaluated proxy-report questionnaires for children aged $<5$ years were included, in line with our inclusion criteria.

\section{Data extraction}

For all eligible studies, two independent reviewers (JA and either JG or AV) extracted data using a structured form. Disagreements were resolved through discussion. The following data was extracted: evaluated measurement properties, study population (i.e., population included in study), target population (i.e., population for which the questionnaire was developed), measurement instrument (i.e., name, construct(s), format), respondent, recall period, comparison method (in case of validity), 
time interval (in case of test-retest reliability), statistical method used, and results of the examined measurement properties (i.e., reliability, validity, responsiveness).

\section{Methodological quality assessment}

Risk of bias was assessed by rating the methodological quality of included studies, using the COnsensusbased Standards for the selection of health Measurement Instruments (COSMIN) checklist [28]. Risk of bias refers to whether results are trustworthy based on the methodological quality of the study. For each examined measurement property, the study design requirements were rated as either very good, adequate, doubtful, or inadequate quality [28]. The lowest score counts method was applied, e.g., if one item was rated inadequate, the final methodological quality was rated as inadequate. For the rating of construct validity studies, the measurement properties of the comparator instrument(s) had to be taken into account. Measurement properties of accelerometers were evaluated using the systematic review of Lettink et al. [29]. An overview of the methodological quality ratings that could be given to different comparator instruments can be found in Additional file 2.

Two independent reviewers (JA and either TA, MC or $\mathrm{AL}$ ) rated the methodological quality of the included studies. In the case of disagreement, a third researcher was consulted (either TA or MC).

\section{Rating study results}

The study results of each measurement property were rated against the criteria for good measurement properties proposed in the COSMIN guideline (i.e., sufficient $(+)$, insufficient $(-)$, inconsistent $( \pm)$ or intermediate (?)) [30]. Below is indicated for each measurement property which outcomes were considered sufficient $(+)$. Outcomes were considered insufficient $(-)$ when these criteria were not met, and intermediate (?) when not all necessary information was reported. Inconsistent $( \pm)$ outcomes were only applicable for validity and reliability studies reporting multiple outcomes per questionnaire, and were rated as described below. The COSMIN methodology for evaluating content validity was used to rate the results of content validity studies [31]. For none of the included (versions of) questionnaires multiple studies on a measurement property were available. For this reason, quantitatively pooling or qualitatively summarizing study results was not possible.

\section{Content validity}

Content validity is defined as "the degree to which the content of a measurement instrument is an adequate reflection of the construct to be measured" [32]. Content validity consists of three components: relevance (e.g., relevant for construct and target population of interest), comprehensiveness (e.g., all key concepts are included), and comprehensibility (e.g., items, response options, and recall period are understood by the population of interest as intended) [31]. Relevance, comprehensiveness, and comprehensibility were rated using the criteria for good content validity [31]. All three components had to be rated as sufficient, in order to rate the overall content validity as sufficient [31].

\section{Internal structure: structural validity, internal consistency, and cross-cultural validity}

Internal structure refers to how the different items in a questionnaire are related, which is important to know for deciding how items might be combined into a scale or subscale [32]. To examine internal structure, three different measurement properties should be evaluated: (1) structural validity, (2) internal consistency and (3) cross-cultural validity. Structural validity and/or internal consistency are only applicable to questionnaires that represent a reflective measurement model, i.e., in which the items of the questionnaire are a reflection of the construct to be measured [27]. In a reflective model, the items are supposed to be correlated and interchangeable [26]. As a reflective measurement model is only applicable to questionnaires assessing sleep quality (i.e., items are a reflection of the construct sleep quality), structural validity and/or internal consistency were only evaluated for questionnaires assessing sleep quality.

Structural validity is defined as "the degree to which the scores of a questionnaire are an adequate reflection of the dimensionality of the construct to be measured" [32], and is usually evaluated by factor analysis. In case of exploratory factor analysis, structural validity outcomes were considered sufficient when factor loadings were $\geq 0.30$ [33]. In case of confirmatory factor analyses, structural validity outcomes were considered sufficient when the comparative fit index or Tucker-Lewis index was $>0.95$, mean square error of approximation was $<0.06$, or standardized root mean residual was $<0.08$ [30, 34].

Internal consistency refers to "the degree of the interrelatedness among the items", and is often evaluated by Cronbach's alpha [28, 32]. Internal consistency outcomes were considered sufficient if Cronbach's alpha values were $\geq 0.70$ and at least low quality of evidence for sufficient structural validity was provided (as rated by COSMIN guidelines) [30].

Cross-cultural validity or measurement invariance is defined as "the degree to which performance of the items of a translated or culturally adapted questionnaire are an adequate reflection of the performance of the items of an original version of the questionnaire" [32]. Crosscultural validity is evaluated by group factor analyses or 
differential item functioning (DIF). When no important differences were found between group factors or DIF for group factors, cross-cultural validity/measurement invariance was considered sufficient [30].

\section{Reliability}

Reliability is defined as "the degree to which the measurement is free from measurement error" [32]. Reliability outcomes were considered sufficient if the intraclass correlation coefficients (ICC) or Kappa (K) values were $\geq 0.70$ [30]. When Pearson or Spearman correlations were used to assess reliability, correlation coefficients had to be $\geq 0.80$, because these correlations do not take systematic errors into account [26]. Most studies reported multiple correlations per questionnaire for test-retest reliability, e.g., separate correlations for each question or item. For this reason, an overall questionnaire rating was applied, i.e., incorporating all correlations, in order to obtain a final test-retest reliability rating for each questionnaire. When $\geq 75 \%$ of correlations were acceptable, a sufficient $(+)$ rating was received, when $\geq 50 \%$ and $<75 \%$ of correlations were acceptable an inconsistent $( \pm)$ rating was received, and an insufficient (-) rating was received when $<50 \%$ of correlations were acceptable.

\section{Measurement error}

Measurement error is "the systematic and random error of a score that is not attributed to true changes in the construct to be measured" [32]. Measurement error outcomes were considered sufficient when the standard error of measurement (SEM), smallest detectable change (SDC) (i.e., defined as "the smallest change that can be detected by the instrument, beyond measurement error" [26]) or limits of agreement (LoA) were smaller than the minimal important change (MIC) (i.e., defined as "the smallest change in score in the construct to be measured that is perceived as important by clinicians or relevant others" [26]) [30]. As the MIC was not defined, we could not give a final rating of the measurement error. Instead, we interpreted the measurement error outcomes per study.

\section{Criterion and construct validity}

Criterion validity is defined as "the degree to which the scores of a measurement instrument are an adequate reflection of a gold standard". Criterion validity was considered sufficient when correlations with the gold standard were $\geq 0.70$ [32]. We considered doubly labeled water as a reasonable gold standard for questionnaires aiming to assess physical activity energy expenditure. In addition, we considered polysomnography as a gold standard for questionnaires assessing sleep. Other comparators (e.g., accelerometers, diaries) were considered to reflect construct validity. Construct validity is "the degree to which the scores of a measurement instrument are consistent with (a priori drafted) hypotheses" (e.g., with regard to relationships to scores of other instruments, or differences between relevant groups) [32]. Since a priori drafted hypotheses for construct validity were often lacking, we formulated criteria with regard to the relationships with other instruments (e.g., accelerometers). Table 1 provides an overview of the criteria for evaluating the results of construct validity studies. These criteria were in line with previous reviews $[20,21]$, and are based on the similarity of the construct that is measured [30]. Additionally, we formulated criteria for studies that evaluated construct validity by comparing subgroups (i.e., children with and without sleep problems). The criteria were subdivided by level of evidence, level 1 indicating strong evidence, level 2 indicating moderate evidence, and level 3 indicating weak evidence. These levels of evidence indicate the confidence in the comparison method to accurately assess the relevant construct. Most studies reported multiple correlations with a comparator instrument, therefore, the same overall rating as used for reliability was applied for each questionnaire (i.e., sufficient $(+)$, inconsistent $( \pm)$, or insufficient $(-))$.

\section{Responsiveness}

Responsiveness is "the ability of a measurement instrument to detect change over time in the construct to be measured" [32]. As none of the included studies evaluated responsiveness of the questionnaire under study, responsiveness is not reported.

\section{Quality of evidence grading}

The quality of evidence was graded using the Grading of Recommendations Assessment, Development and Evaluation (GRADE) approach, as proposed in the COSMIN guideline [30], indicating the trustworthiness of the measurement property results of questionnaires. The GRADE approach consists of four levels (i.e., high, moderate, low, very low), which depend on the presence of four risk factors: (1) risk of bias (i.e., the methodological quality of the studies), (2) inconsistency (i.e., unexplained inconsistency of results across studies), (3) imprecision (i.e., total sample size of the available studies), and (4) indirectness (i.e., evidence from different populations than the population of interest in this review) [30]. The quality of evidence was subsequently downgraded with one, two or three levels for each factor to moderate, low, or very low when there was risk of bias, inconsistency in results, a low sample size, or indirect results [30]. Because the COSMIN methodology has been updated since publication of the previous reviews, we re-evaluated all included studies from previous reviews 


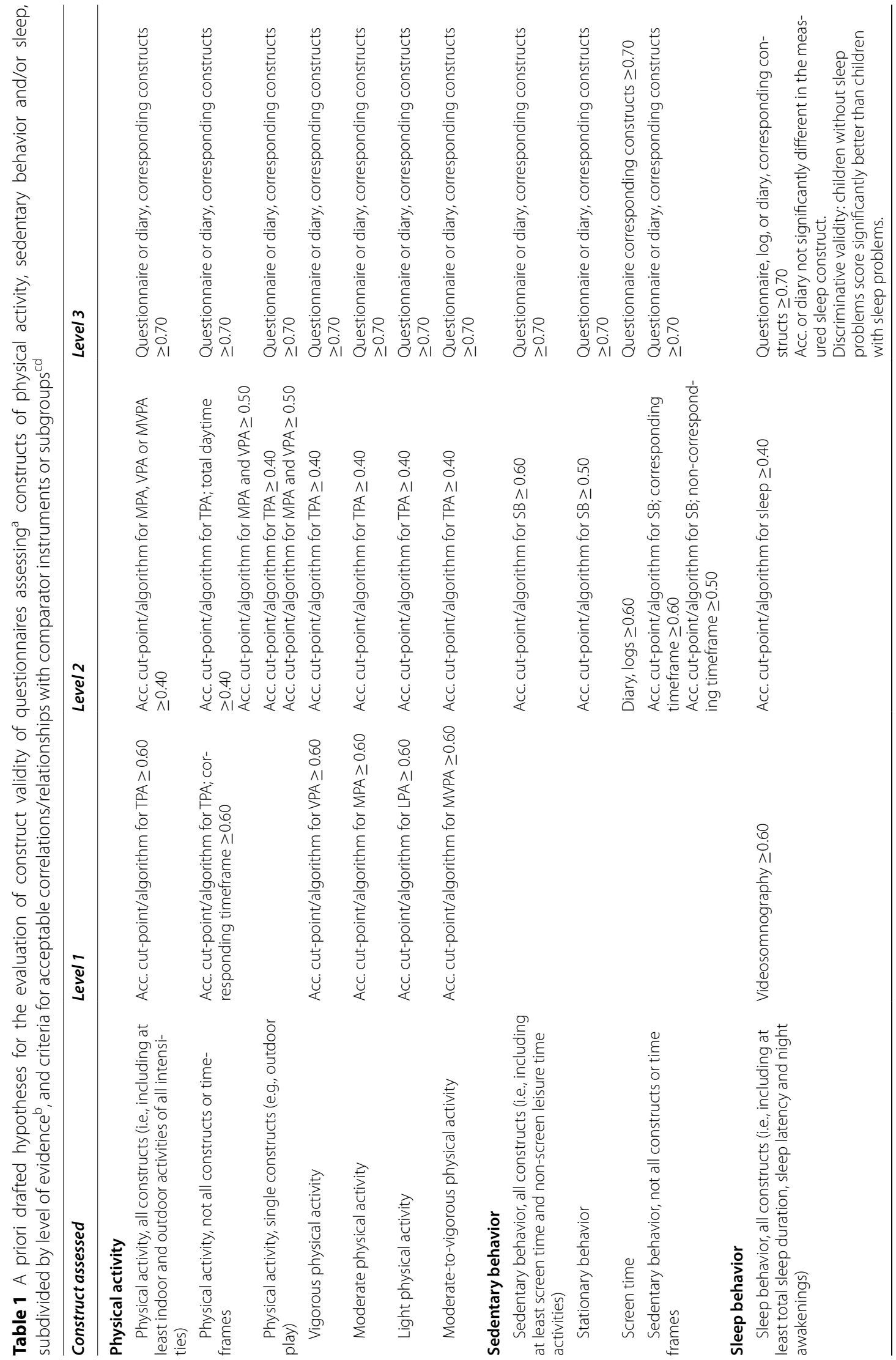




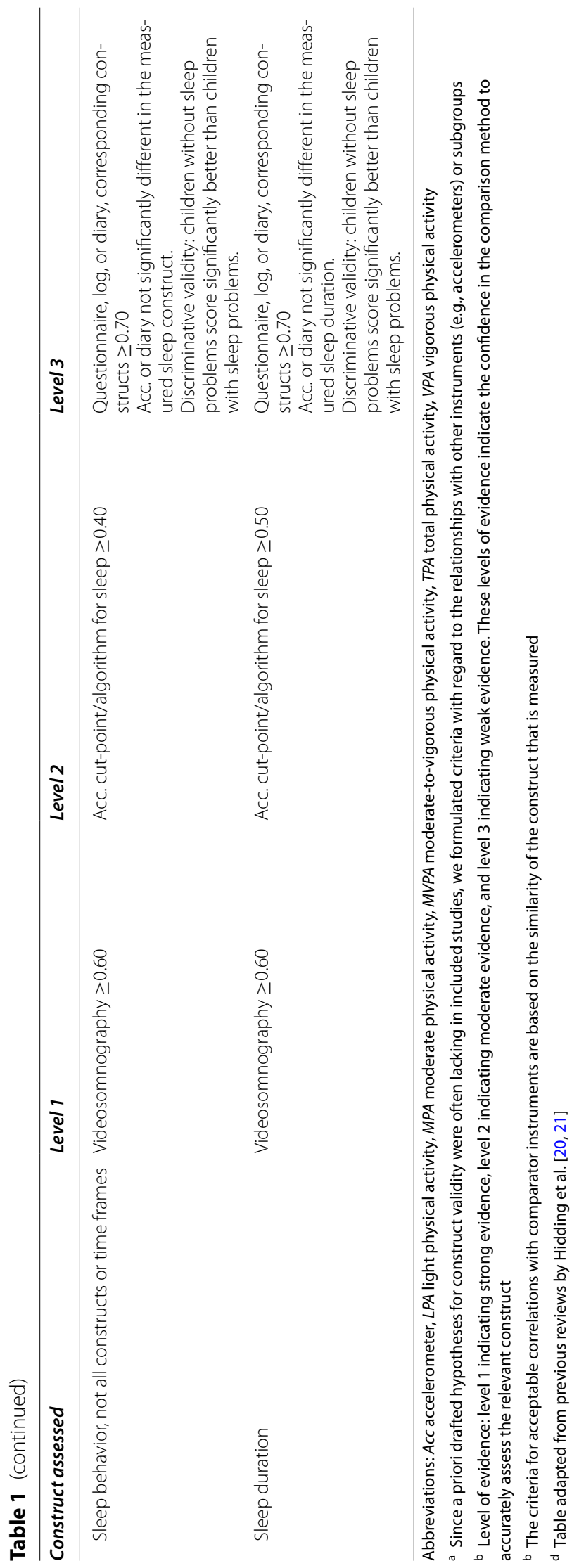


on methodological quality and regraded the quality of evidence [30]. The grading of the quality of evidence was done for each measurement property and for each questionnaire separately by one researcher (JA). The ratings of measurement property outcomes (i.e., sufficient, insufficient, inconsistent, intermediate) were only presented in the results section for the measurement properties of questionnaires that received a high or moderate quality of evidence grading, as these results are considered most trustworthy. Consequently, a questionnaire can only be considered as valid/reliable when the quality of evidence is at least moderate, and the reliability/validity results are sufficient.

\section{Results}

Systematic literature searches using the PubMed, Embase, and SPORTDiscus databases yielded a total of 12,390 unique articles. After title and abstract screening, 46 full-texts were screened. Additionally, 14 articles were found through cross-reference searches. Therefore, a total of 60 full-text articles were assessed for eligibility, of which 25 were included. Additionally, we included 8 relevant articles from previous reviews [19-21], resulting in 33 articles that evaluated a total of 37 different (versions of) questionnaires (see Fig. 1 for the full selection process). Four questionnaires were (translated) versions of the Brief Infant Sleep Questionnaire (BISQ) [35-38], and three questionnaires were versions of the Children's Sleep Habits Questionnaire (CSHQ) [39-41]. Table 2 presents the characteristics of all included questionnaires. Tables 3-6 summarize the results for content validity, internal consistency and structural validity, reliability, and criterion validity and construct validity.

\section{Description of questionnaires}

Of the included questionnaires, 10 were designed for infants specifically ( $0-1$ year old). These questionnaires all assessed constructs of sleep [37-39, 42-45]. Two questionnaires were designed for toddlers (1-3years old), of which one assessed sleep [47], and one screen behavior [53]. Eleven questionnaires were designed for preschoolers (3-5 years old). Five of these questionnaires assessed constructs of sleep [40,41,48-50], two physical activity and sedentary behavior [58, 59], and four assessed constructs of all 24-h movement behaviors [62, $63,66,67]$. Fourteen questionnaires were designed for multiple of the aforementioned age groups. Three questionnaires assessed sleep behavior in infants and toddlers $[35,36,46]$. Two questionnaires targeted both infants, toddlers, and preschoolers, of which one assessed screen behavior [52], and one physical activity and sedentary behavior [64]. Nine questionnaires targeted toddlers and preschoolers, of which one assessed sleep behavior [51], four assessed physical activity $[54,55]$, two assessed constructs of both physical activity and sedentary behavior $[56,57]$, and two assessed constructs of all 24-h movement behaviors $[60,61,65]$.

Respondents of the questionnaires were parents or caregivers, except for two questionnaires that were completed by family child care providers, i.e., the modified Burdette proxy report and the modified Harro proxy report [55]. Recall periods varied across questionnaires, ranging from current day $(n=9)$ to a typical week $(n=3)$, with a typical (week or weekend) day being used most frequently $(n=13)$. Four questionnaires used ordinal response options (e.g., Likert scale), 17 continuous (e.g., duration in hours and/or minutes), one nominal and 14 questionnaires used a combination of these response options.

\section{Content validity}

Six studies reported data on the comprehensiveness, comprehensibility and/or relevance of the items of the questionnaire under study (Table 3). Two of the examined questionnaires assessed constructs of sleep behavior, i.e., the Children Sleep Wake Scale (CSWS) [51] and Nepali version of the BISQ [36]. One questionnaire assessed screen behavior in children aged 0-5, i.e., the Technology Use Questionnaire (TechQ-U) [52]. The other three questionnaires, i.e., the Healthy Kids [60], the Family Health Survey [66], and the Surveillance of digital Media habits in early childhood Questionnaire $\left(\mathrm{SMALLQ}^{\mathrm{TM}}\right.$ ) [65], assessed constructs of all 24-h movement behaviors in toddlers and/or preschoolers. Questionnaire development was reported in five studies $[51,52,60,65$, 66]. These studies used cognitive interviews [60, 65, 66], semi-structured interviews [52], focus groups [65], and/ or expert opinions $[51,52,65]$ to evaluate content validity of the questionnaire. Two of these questionnaires were additionally pilot-tested in a small sample of caregivers to provide information on, for example, readability or time to complete the questionnaire [51, 65]. Overall, we graded the quality of evidence for the content validity of these questionnaires as low. Unfortunately, none of the questionnaires were evaluated on all aspects of content validity (i.e., relevance, comprehensiveness, and comprehensibility). For this reason, we could not rate the overall content validity of these questionnaires.

Internal structure: structural validity, internal consistency, and cross-cultural validity

Internal consistency was evaluated for three questionnaires (Table 4), all assessing sleep behavior: the CSWS, Children's Sleep Habits Questionnaire (CSHQ) and Children's Sleep Habits Questionnaire for infants (CSQHI) [39, 40, 51]. Internal consistency was evaluated by 


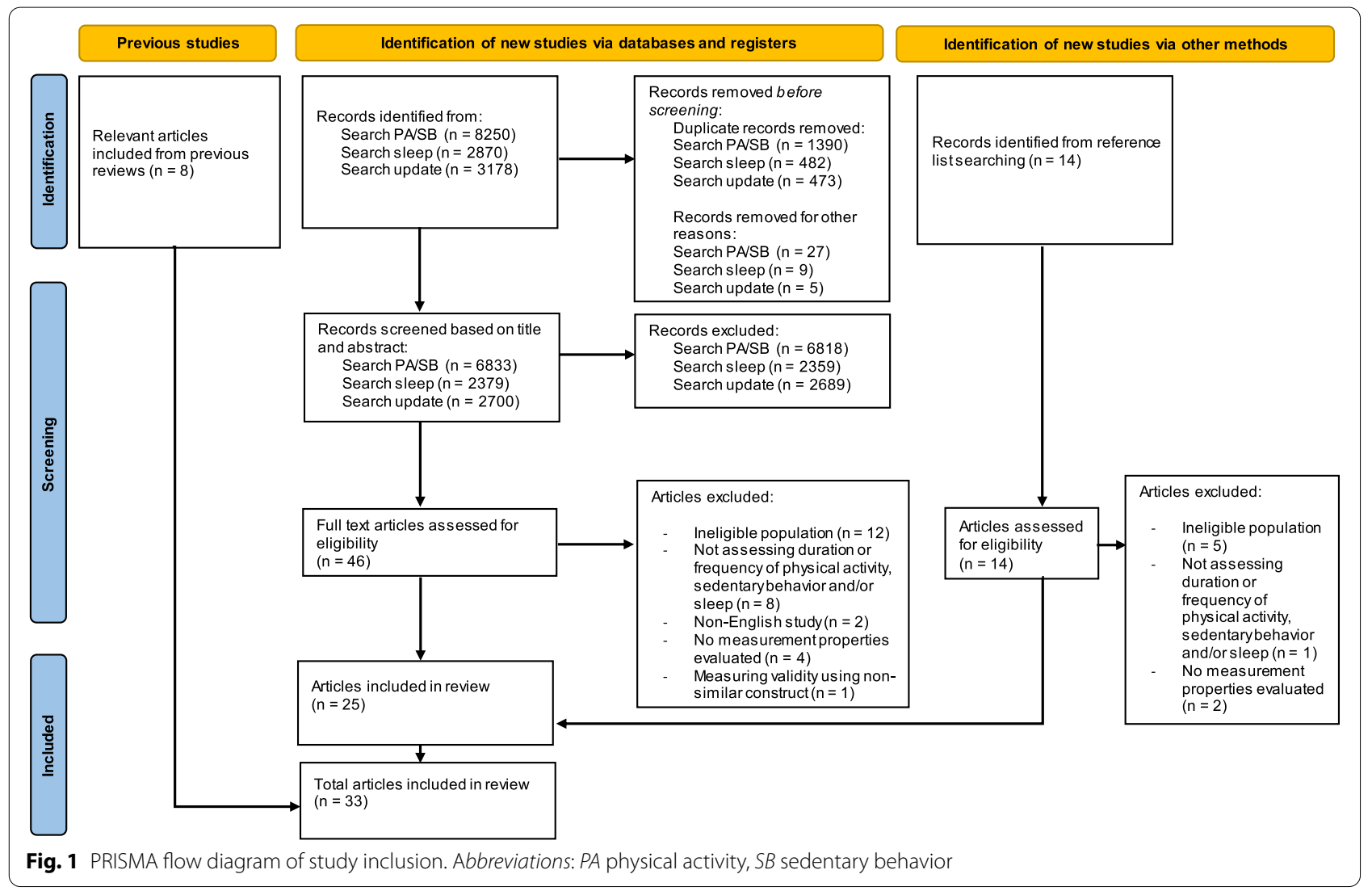

calculating Cronbach's alpha of each subscale for all three questionnaires. The quality of evidence for the internal consistency was high for all three questionnaires. The internal consistency outcomes for the CSWS were rated as sufficient [51], whereas outcomes for the CSHQ and CSQH-I were rated as insufficient [39, 40]. The CSHQ and CSQH-I were also evaluated on structural validity by performing confirmatory factor analysis [40] and/ or exploratory factor analysis $[39,40]$, both receiving a moderate evidence grading (Table 4). Structural validity outcomes of both questionnaires were considered sufficient. None of the translated or culturally adapted questionnaires were evaluated on cross-cultural validity or measurement invariance [35-37, 39, 40, 66].

\section{Reliability}

Sixteen questionnaires were assessed on reliability (Table 5). Six of these questionnaires assessed constructs of sleep [35, 37, 39, 40,45, 51], six assessed constructs of physical activity and/or sedentary behavior [52, 53, 56-59], and four assessed constructs of all 24-h movement behaviors [61-63, 67]. Reliability of nine questionnaires was evaluated by calculating ICC [40, 52, 53, 56-59, 63, 67], whereas Pearson or Spearman correlations were calculated for seven questionnaires $[35,37,39,45,51,61,62]$. Time interval between test and retest ranged between 7 days $[53,57]$ and 3 months [39]. Two questionnaires received a moderate quality of evidence grading for reliability, i.e., the Early Years Physical Activity Questionnaire (EY-PAQ) [57] and Preschool-age Children's Physical Activity Questionnaire (Pre-PAQ) [58]. Reliability outcomes were considered insufficient for both questionnaires. Five questionnaires received a low quality of evidence grading, and nine questionnaires received a very low evidence grading.

\section{Measurement error}

Measurement error was evaluated for one questionnaire, i.e., the Pre-PAQ [58]. The quality of evidence for the measurement error of this questionnaire was graded as moderate. This questionnaire demonstrated a measurement error ranging from 1.0-1.1 min for time spent in organized physical activities. Unfortunately, as the MIC for interpreting the measurement error outcomes was not defined, we could not give a final rating for measurement error. However, a measurement error of 1.0-1.1 min seems acceptable. 


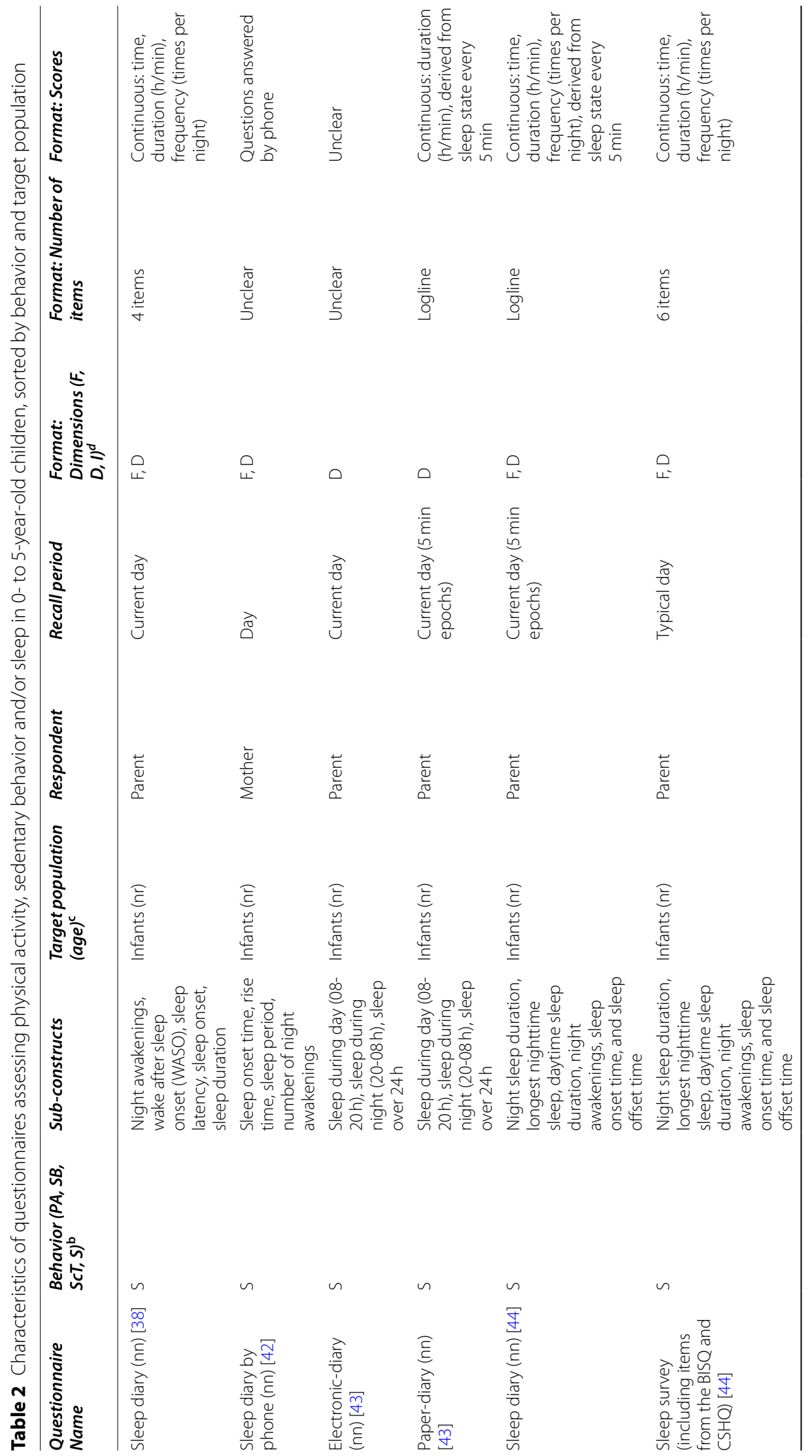




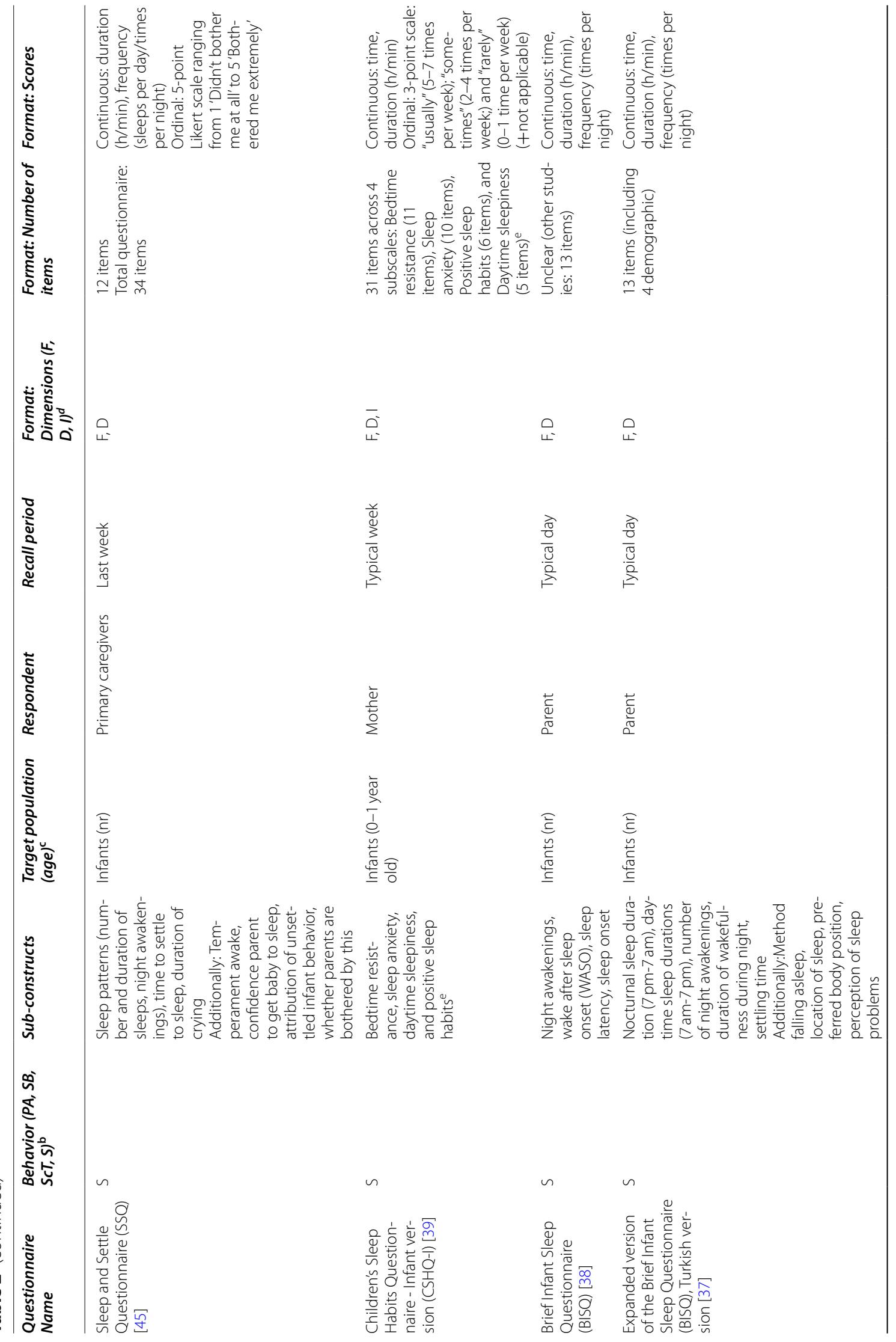




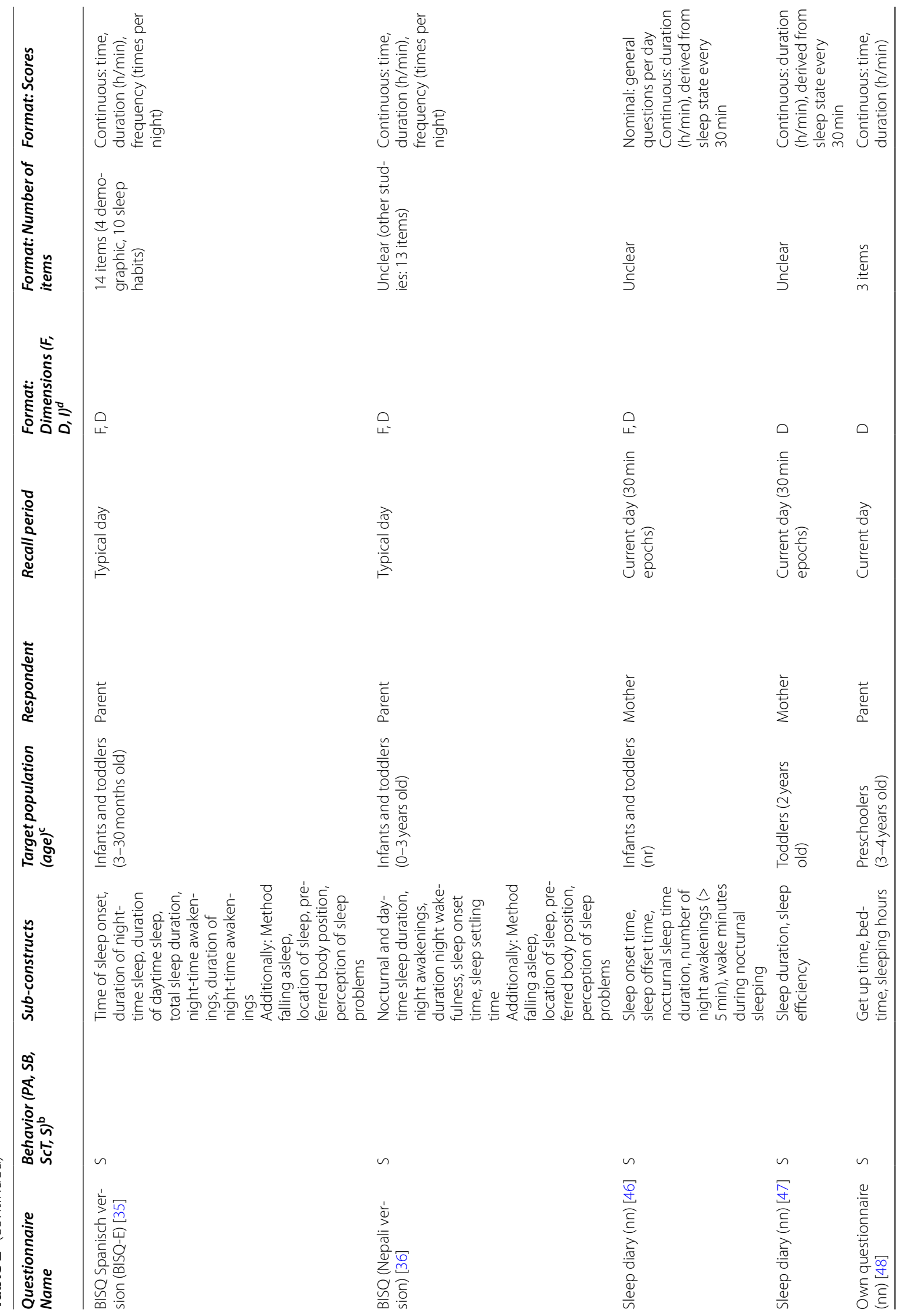




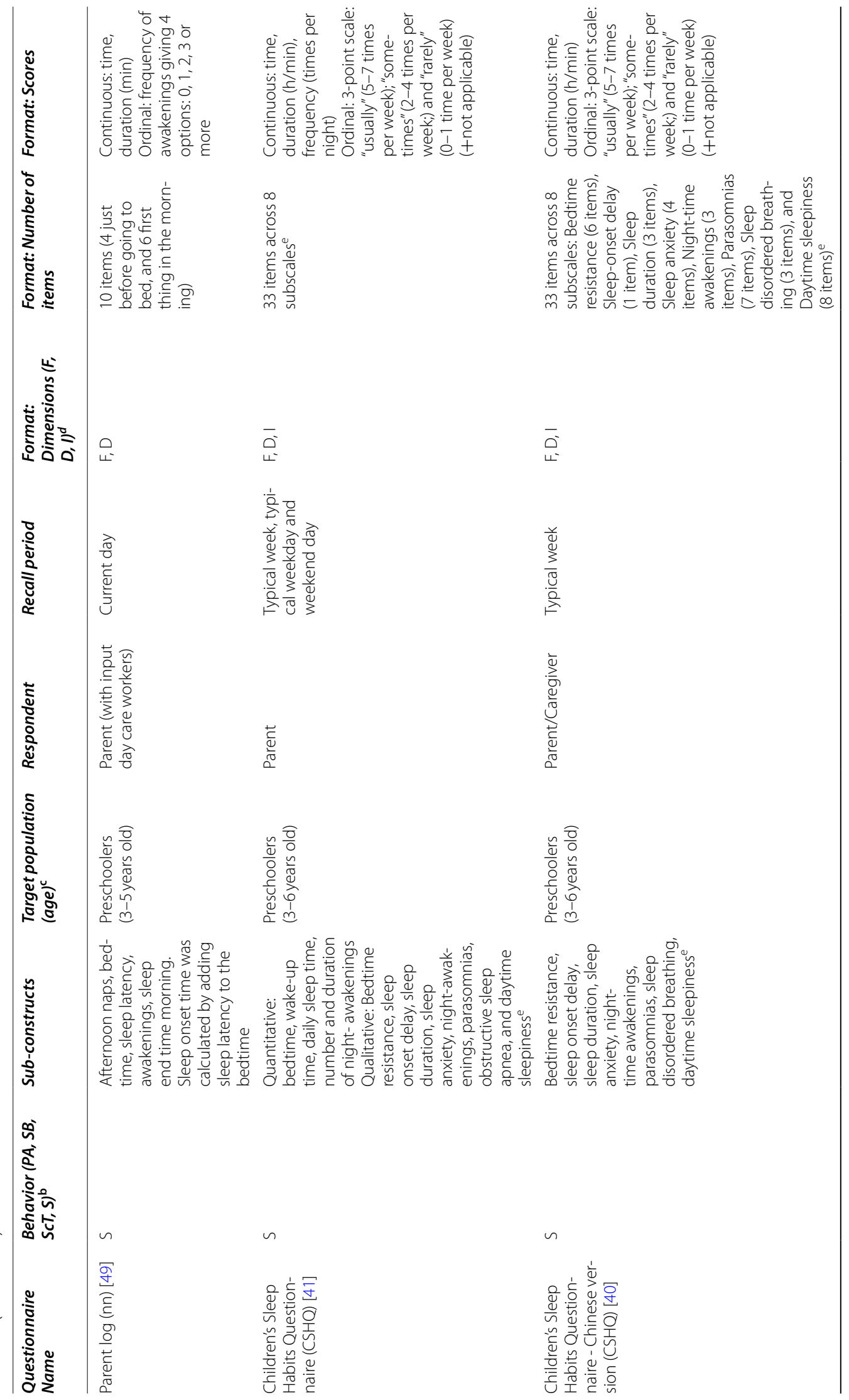




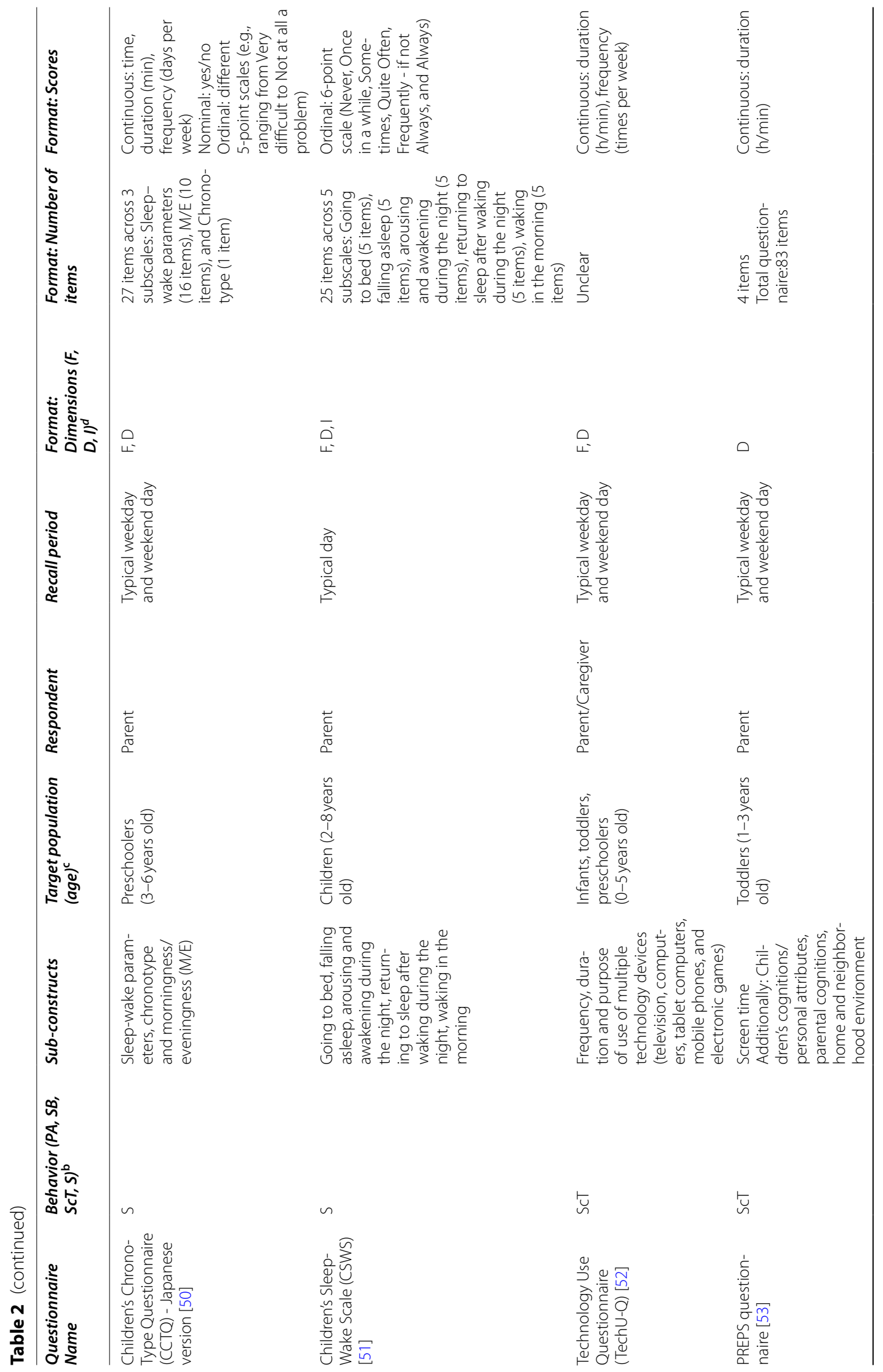




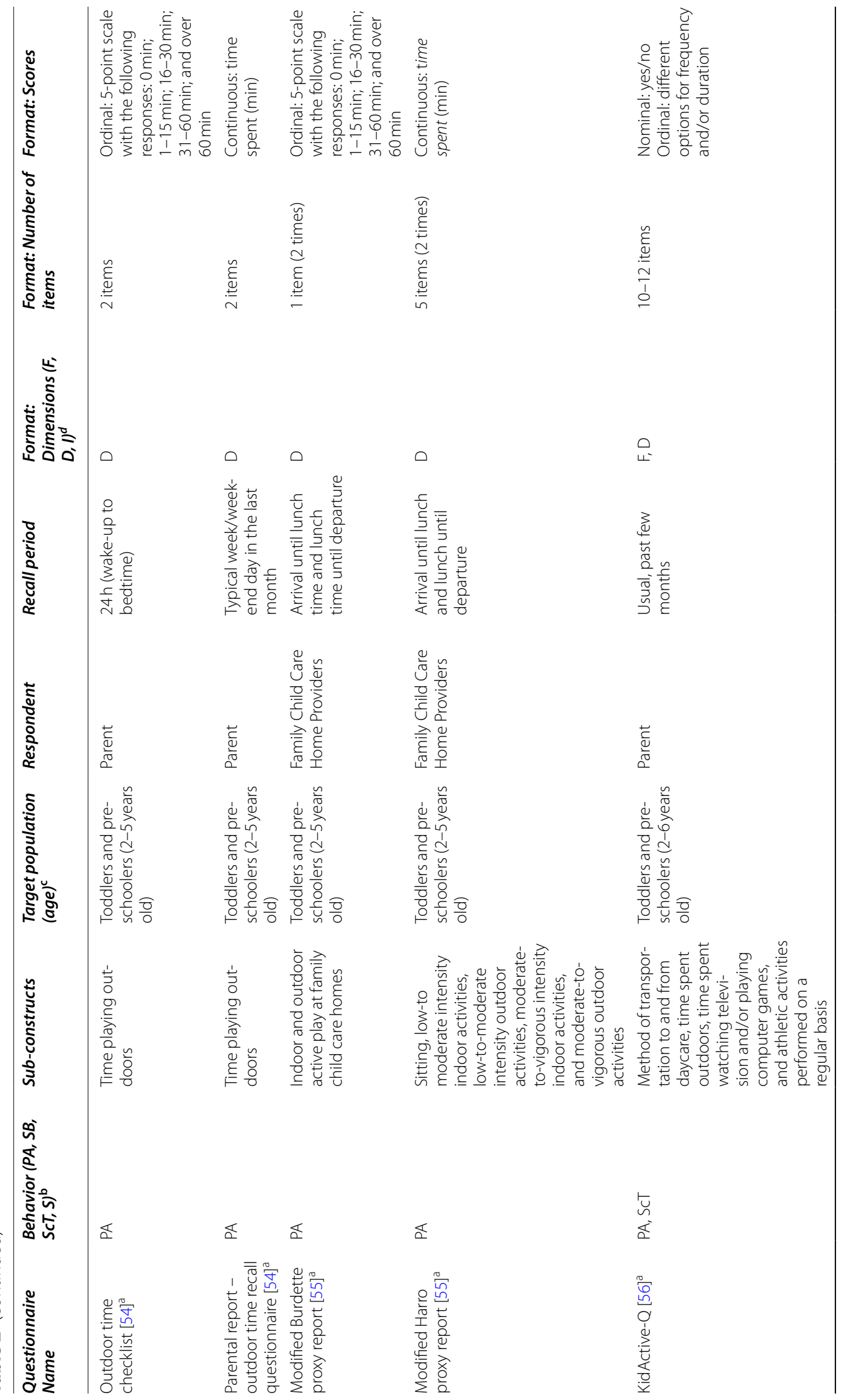




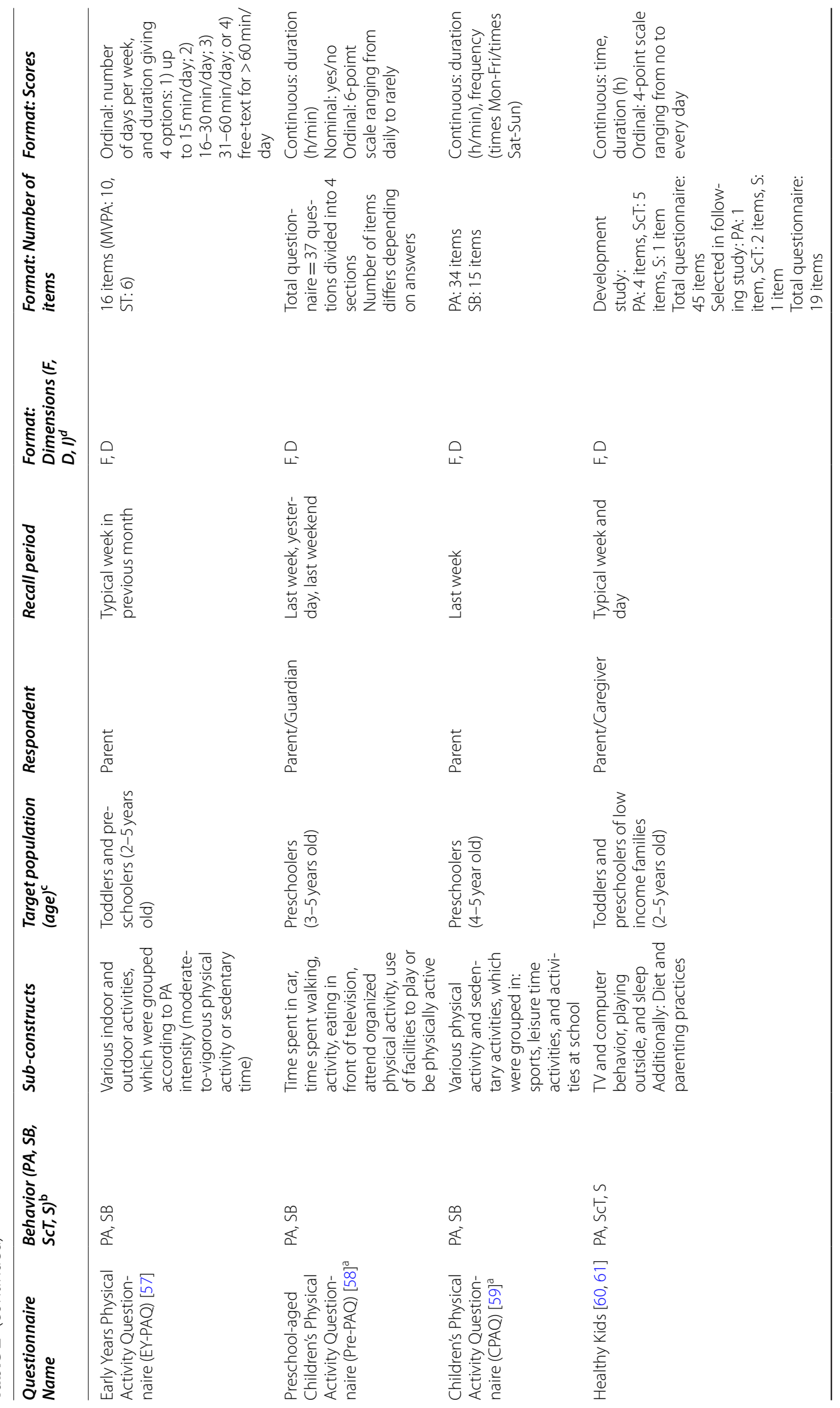




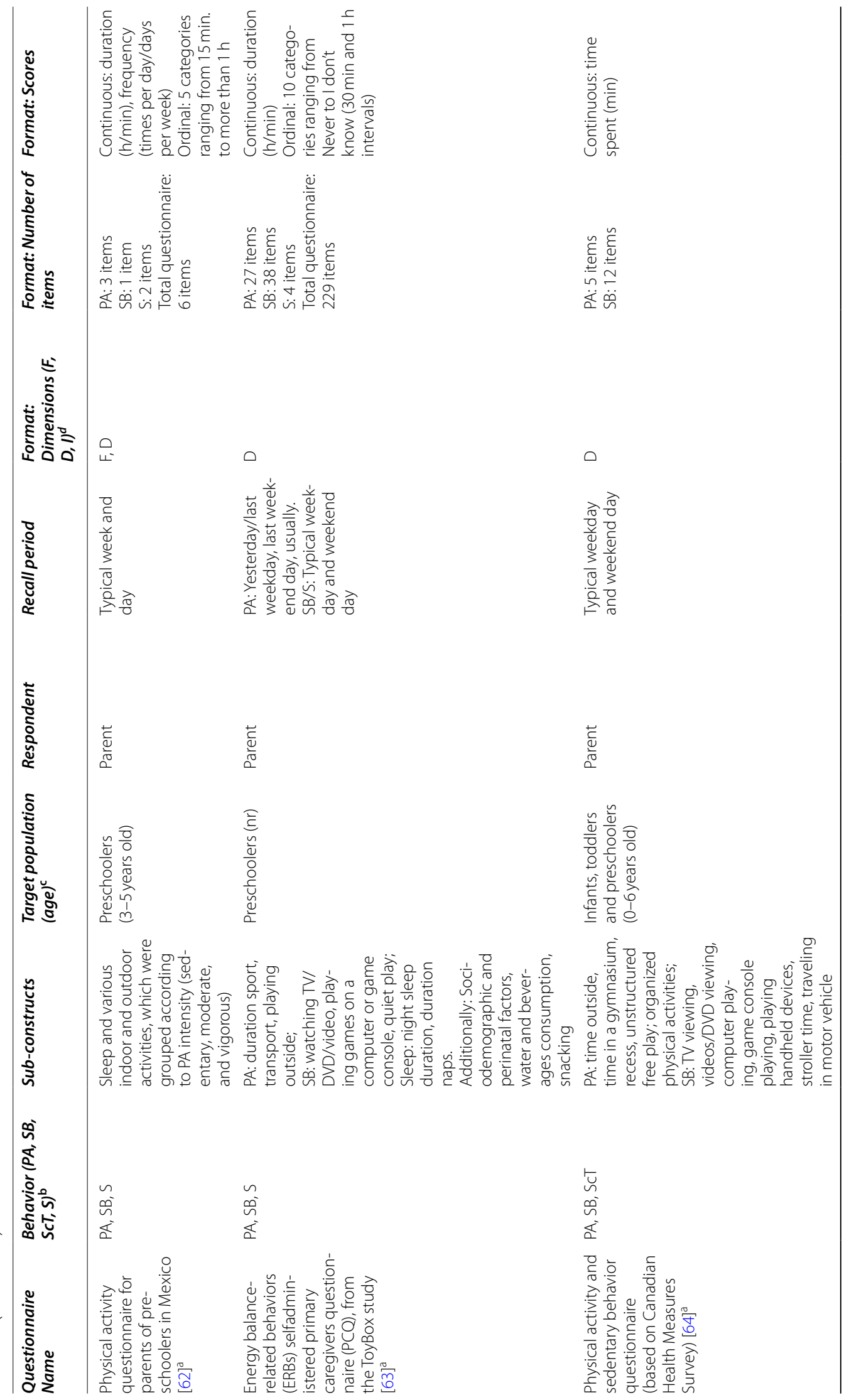




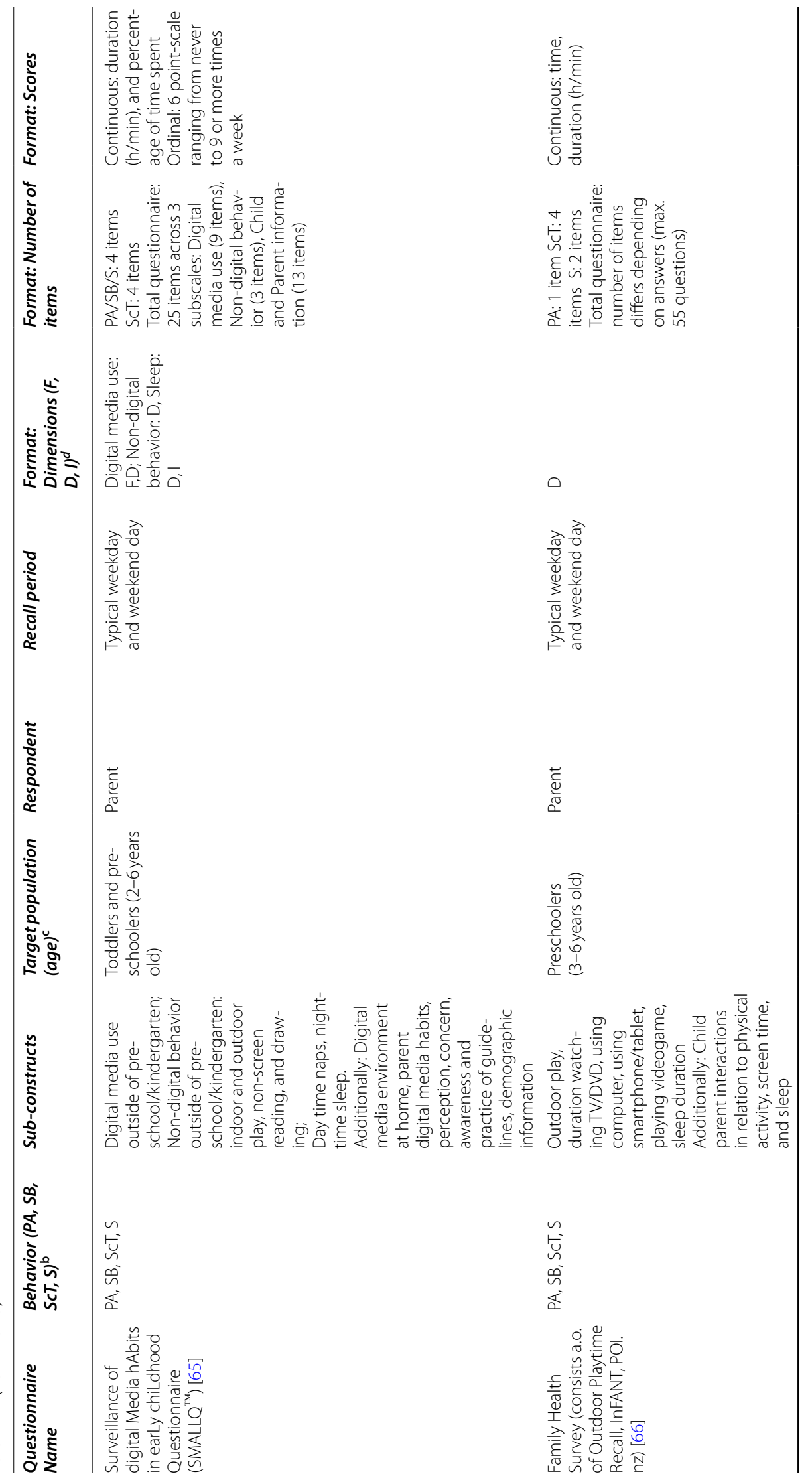




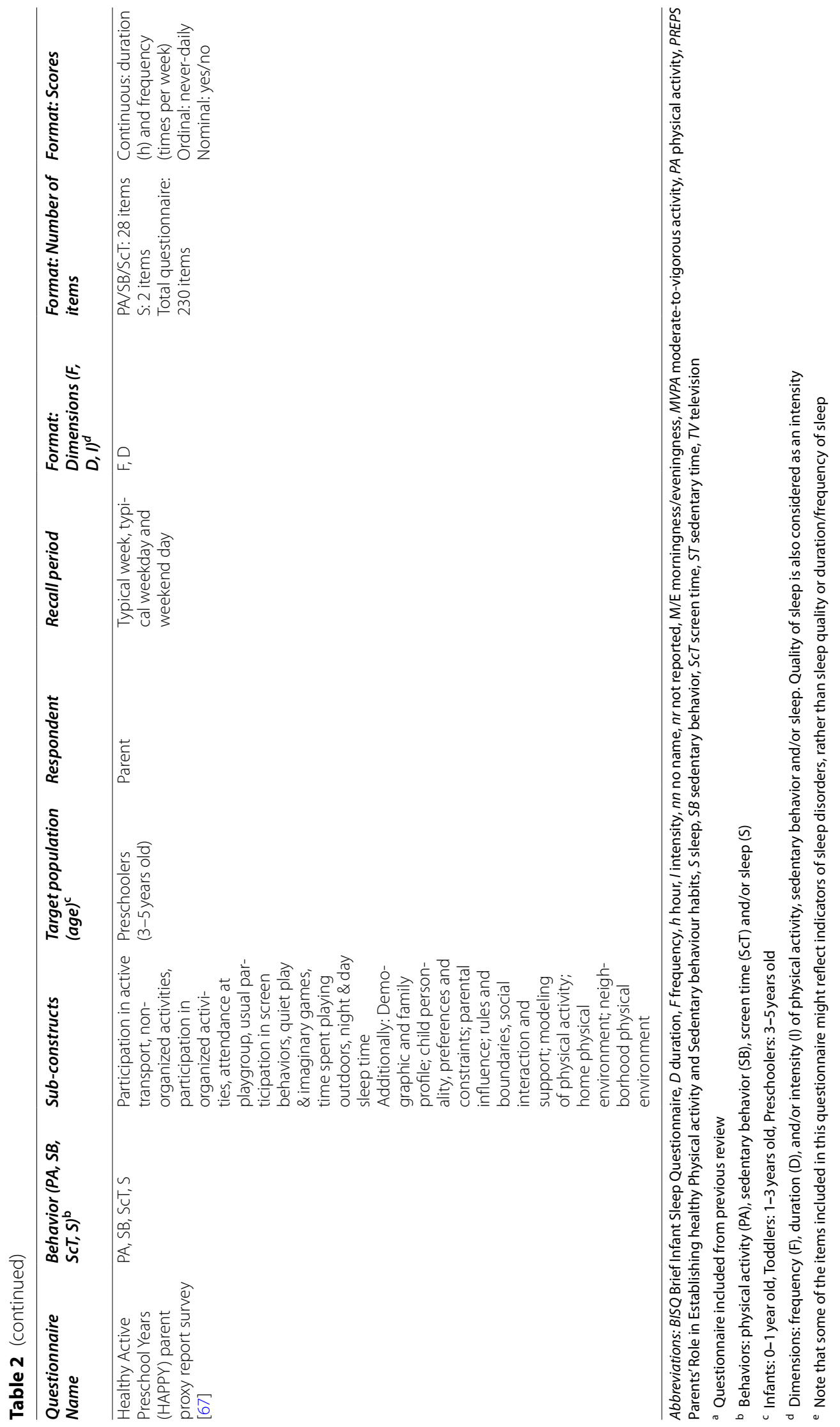




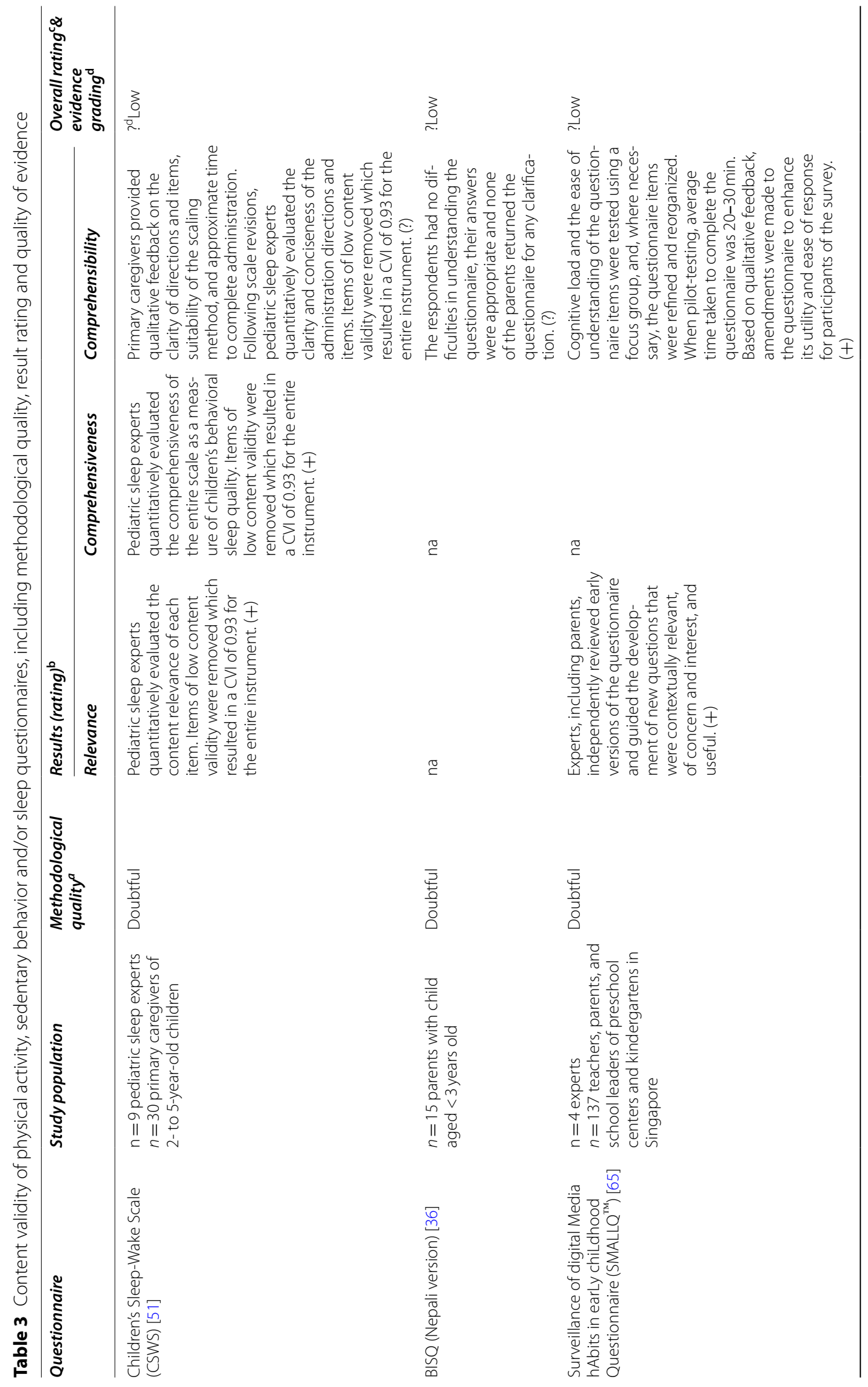




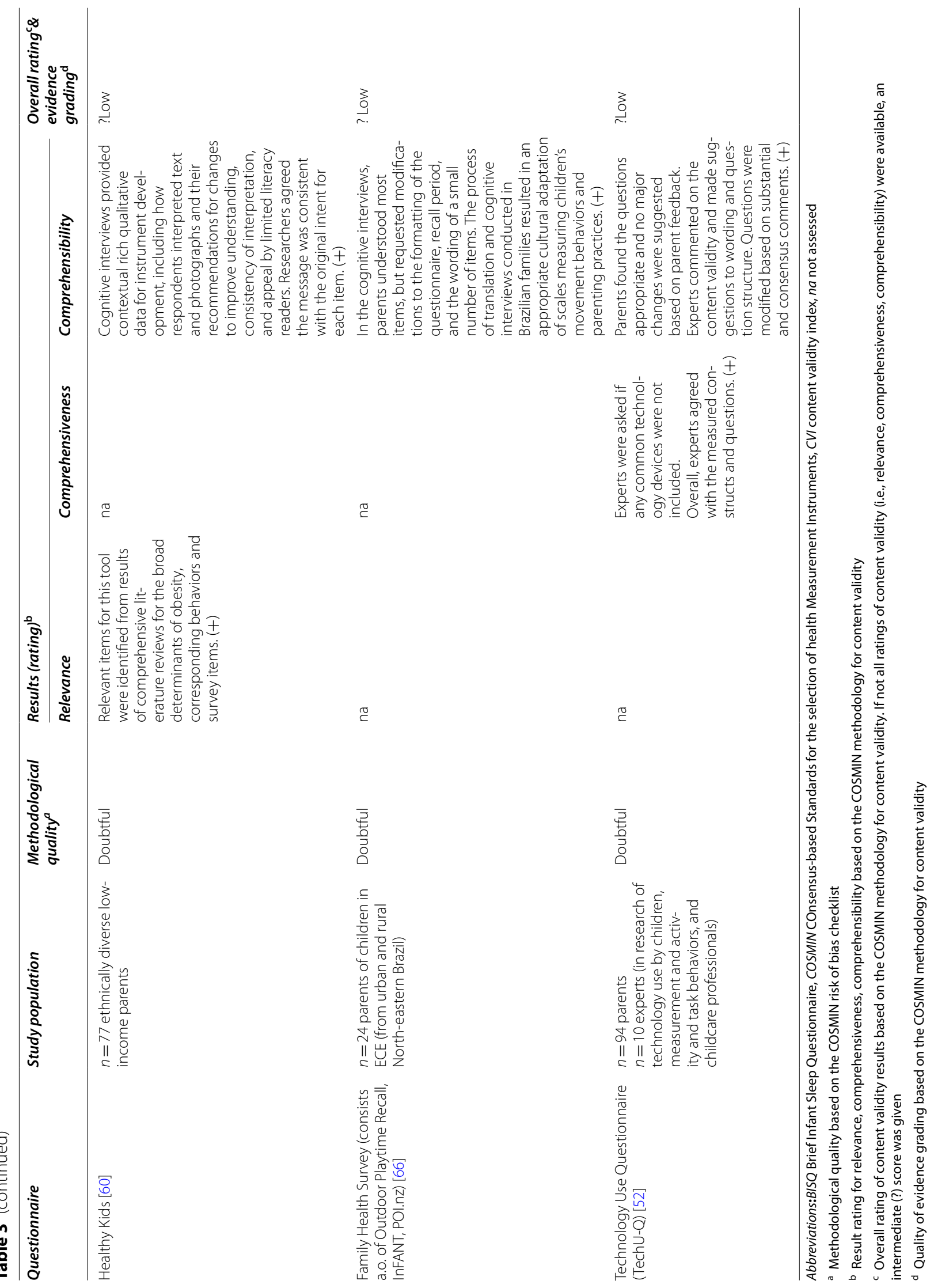




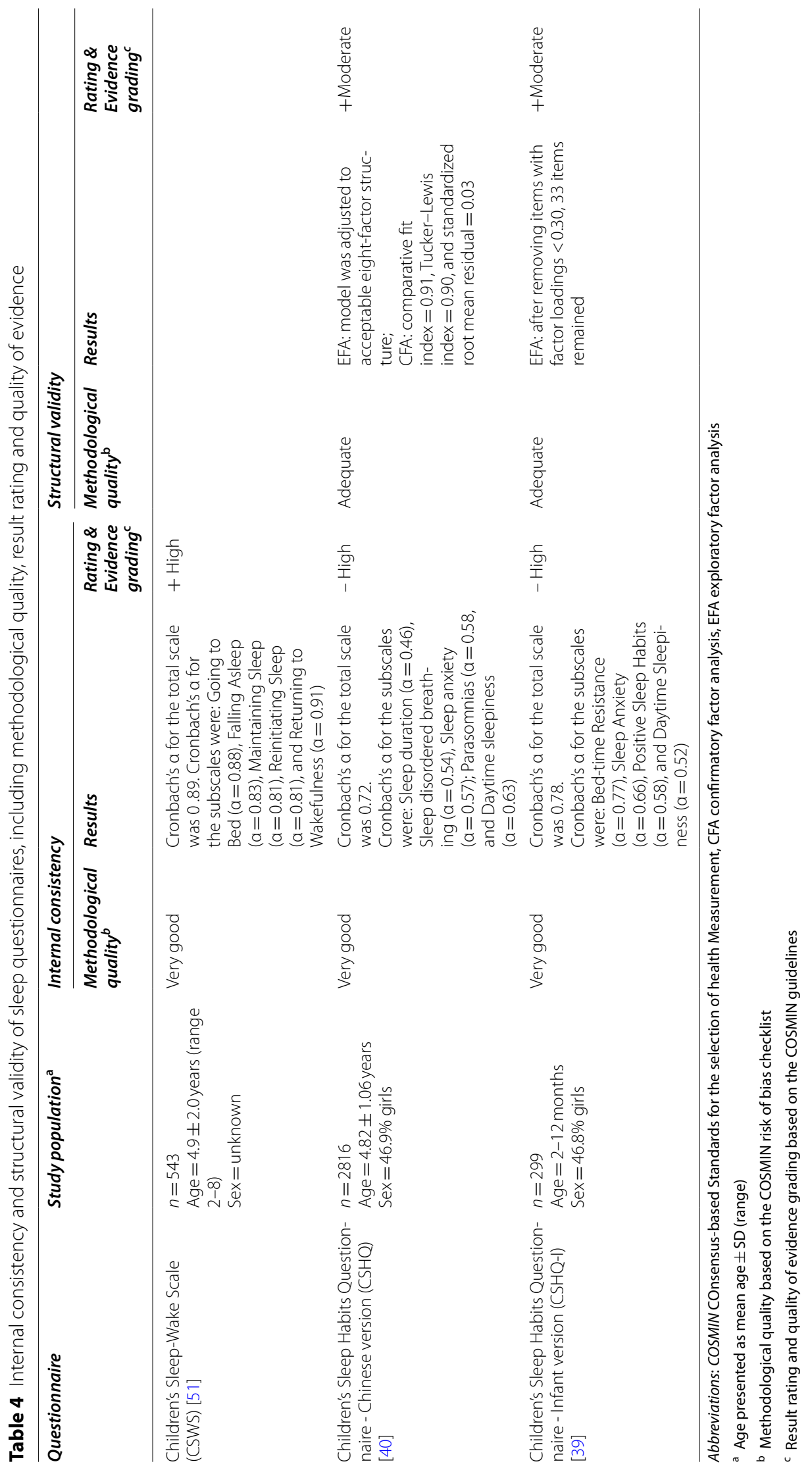




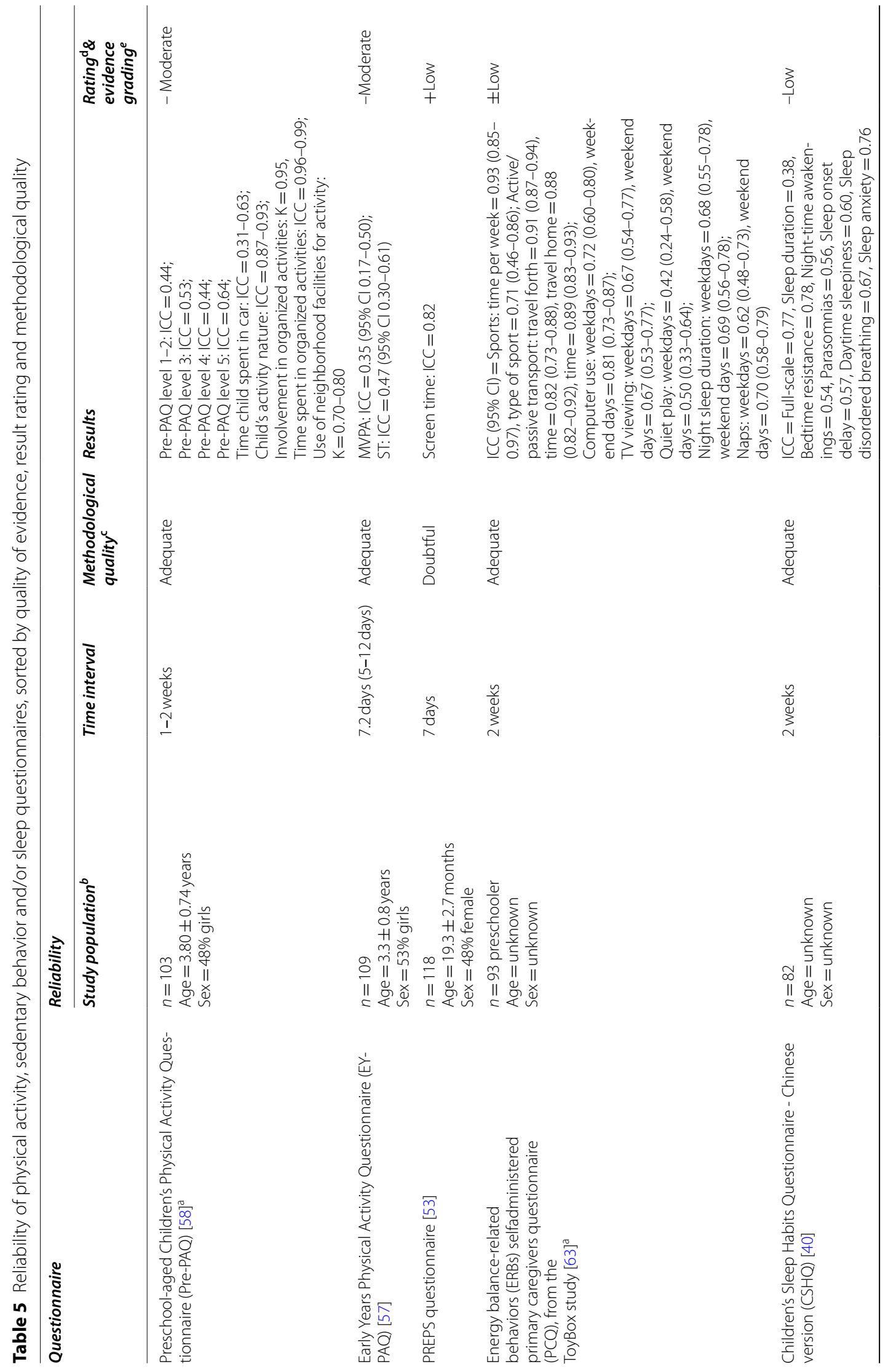


Arts et al. Int J Behav Nut Phys Act <wide> (2022) 19:18

Page 24 of 39

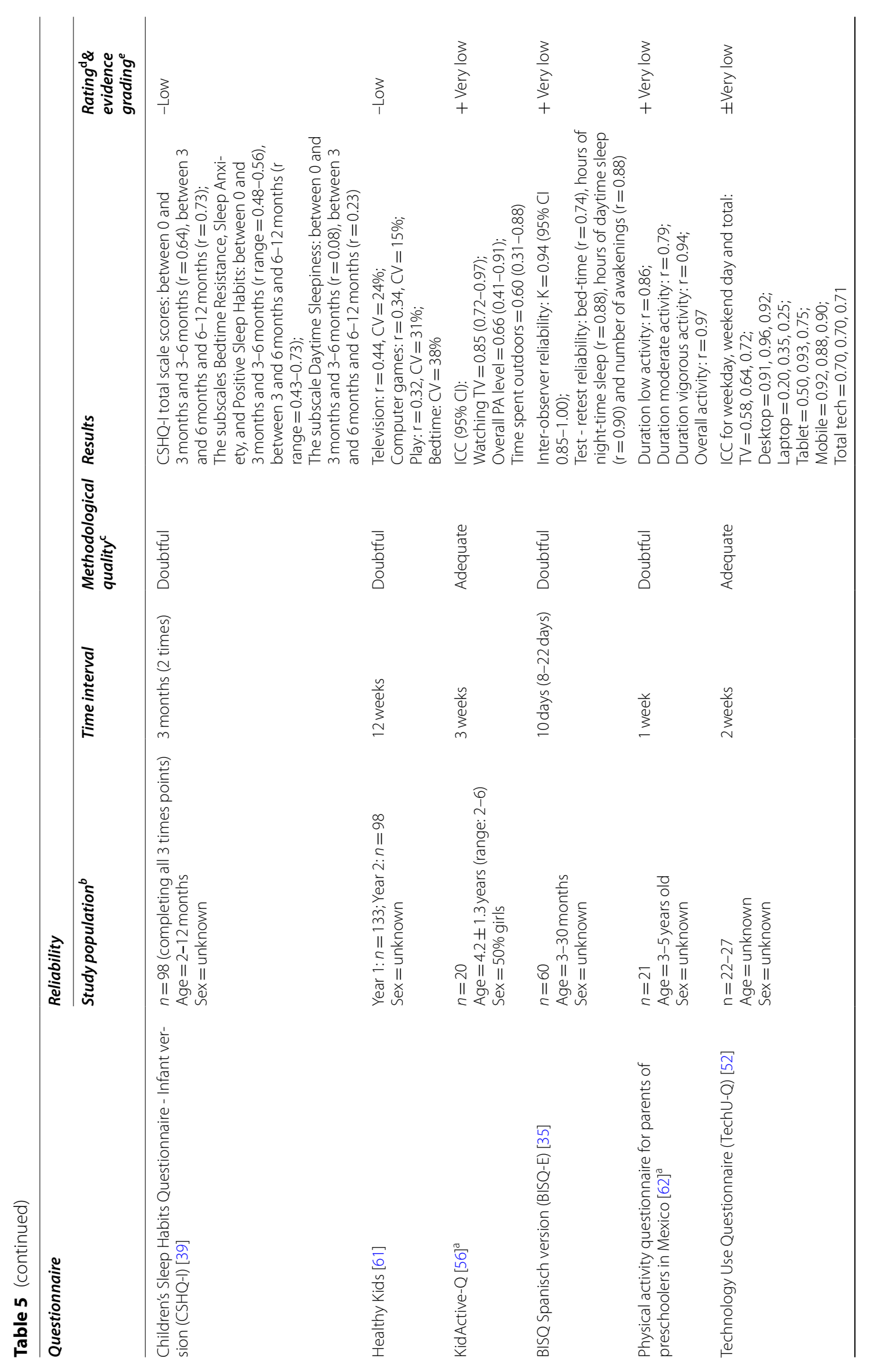




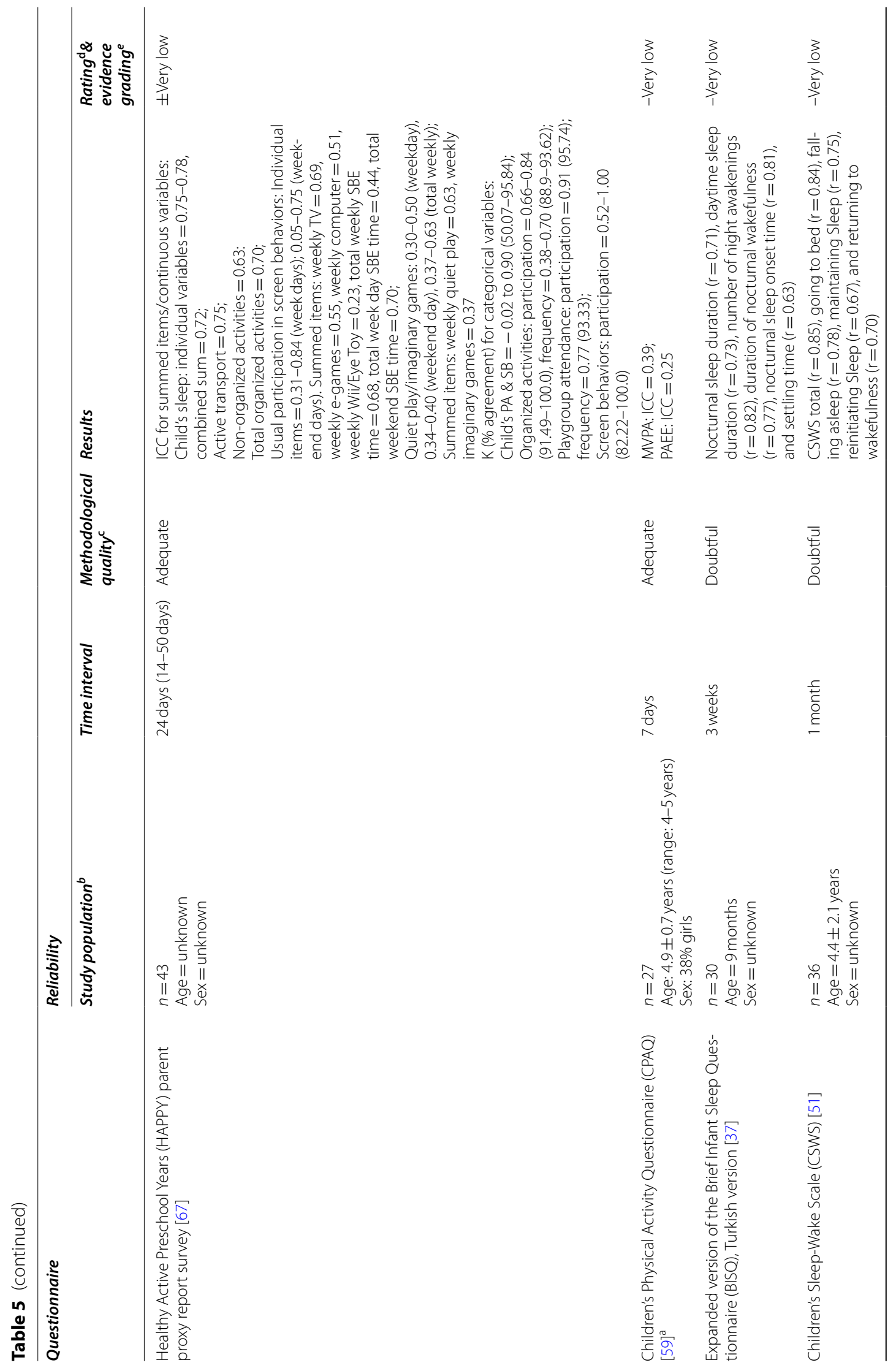




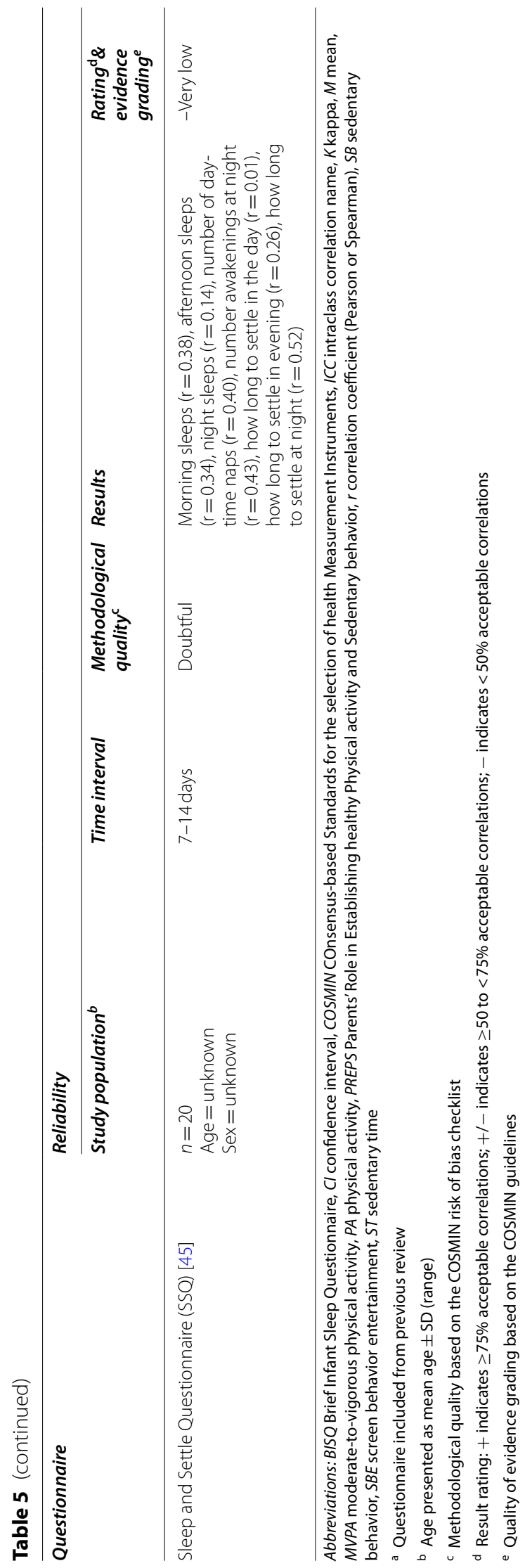




\section{Criterion and construct validity}

Criterion validity was evaluated for one questionnaire (Table 6), i.e., the Children's Physical Activity Questionnaire (CPAQ) [59]. The CPAQ was used to assess energy expenditure in 4- to 5-year-olds. This questionnaire received a low quality of evidence grading for criterion validity, and outcomes were considered insufficient.

Twenty-one studies evaluated the construct validity of a total of 26 different questionnaires (Table 6). Sixteen of these questionnaires assessed constructs of sleep [35, 38-50], eight assessed constructs of physical activity and/or sedentary behavior $[54,55,57-59,64]$, and two assessed constructs of all three movement behaviors [61, 62]. Thirteen studies evaluated construct validity using an objective comparator instrument (e.g., accelerometer) [41-43, 47-49, 55, 57-59, 62, 64], three used a subjective comparator instrument (e.g., diary) [35, 39, 61], and four studies used both [38, 44, 50,54]. Nineteen studies used Pearson or Spearman correlations to evaluate construct validity, of which 10 studies also presented Bland-Altman plots [38, 41-44, 47, 54, 57-59, 64]. One study evaluated construct validity by comparing subgroups (i.e., discriminative validity) [45]. The quality of evidence for construct validity was graded as moderate for one questionnaire, i.e., the EY-PAQ [57], showing outcomes that were considered insufficient. Fifteen of the questionnaires received a low quality of evidence grading, and 10 questionnaires received a very low evidence grading.

\section{Discussion}

This review summarizes studies that evaluated the measurement properties of proxy-report questionnaires for assessing physical activity, sedentary behavior, and/or sleep in 0 - to 5-year-old children. The questionnaires varied in constructs, format, target population, and evaluated measurement properties. Unfortunately, while we identified 37 relevant questionnaires, none were considered valid and/or reliable for assessing one or more movement behaviors in children aged $0-5$ years.

Proxy-report questionnaires for assessing all 24-h movement behaviors in this young age group are scarce. The majority of included questionnaires assessed one type of behavior, of which sleep behavior was assessed most frequently. Only six questionnaires assessed duration and/or frequency of all three behaviors (physical activity, sedentary behavior, and sleep) $[60-63,65,67]$. Unfortunately, these six questionnaires mostly used very few items per movement behavior, which questions the comprehensiveness of the items (e.g., a single item to assess sedentary behavior). Overall, 21 out of 37 questionnaires targeted children above 2 years old (e.g., preschoolers). Questionnaires that targeted solely infants all assessed sleep behavior $(n=10)$. Only one questionnaire (i.e., based on the Canadian Health Measures Survey) assessed physical activity and sedentary behavior in infants, next to toddlers and preschoolers (i.e., 0 - to 6-year-old children) [64]. In addition, one questionnaire assessed screen use in 0 - to 5-year-old children (i.e., TechU-Q) [52]. There were only two questionnaires that specifically targeted toddlers: a questionnaire assessing screen use (i.e., PREPS questionnaire) [53] and a sleep diary [47]. This further indicates the urgent need for developing questionnaires assessing 24-h movement behaviors in infants and toddlers. Notably is the large number of questionnaires $(n=14)$ that were designed for multiple age groups (e.g., toddlers and preschoolers). However, since infants, toddlers and preschoolers each have their own form and context of physical activity, sedentary behavior and sleep [9], tailored questionnaires are needed that fit the specific behavior of the target group.

There are two important issues that complicate drawing definite conclusions regarding the best available questionnaires. First, there is a lack of studies comprehensively evaluating measurement properties of proxyreport questionnaires. These findings are consistent with previous reviews $[20,21,24]$. For example, only one study reported information on the measurement error of a questionnaire [58]. Measurement error is an important characteristic of reliability, as it gives an indication of the systematic and random error expressed in units of measurement (e.g., minutes per day), which facilitates the interpretation of measurements [26]. Unfortunately, our systematic review confirms that measurement error is still largely underreported in studies evaluating measurement instruments [26]. Consequently, we are unable to correctly interpreted the results of research using these questionnaires to assess time spent in physical activity, sedentary behavior and/or sleep. Likewise, criterion validity was evaluated for only one questionnaire [59]. As in many of the included studies the comparison instrument was not considered a gold standard, these studies were considered to reflect construct validity. Additionally, none of the included studies evaluated the responsiveness of the questionnaire. However, for the purpose of longitudinally monitoring one or more movement behaviors, questionnaires should be able to detect changes over time $[26,30]$. Furthermore, none of the translated or adapted questionnaires were evaluated on cross-cultural validity (measurement invariance). Without evaluating this type of validity, it remains unsure if the performance of the translated items are an adequate reflection of the performance of the items of the original questionnaire [30]. Moreover, there is a lack of studies describing the development or content validity of the questionnaire [20, 21]. Only six studies reported information that contributed to 


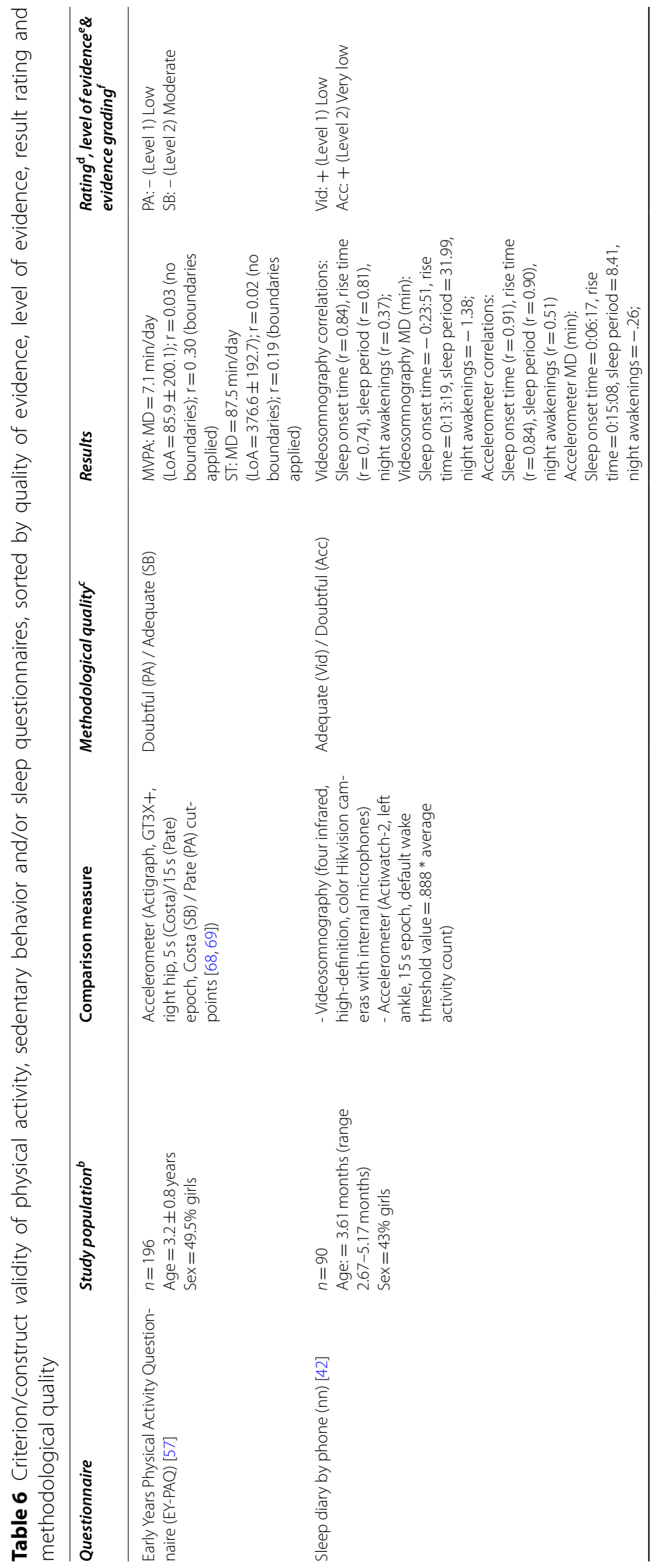




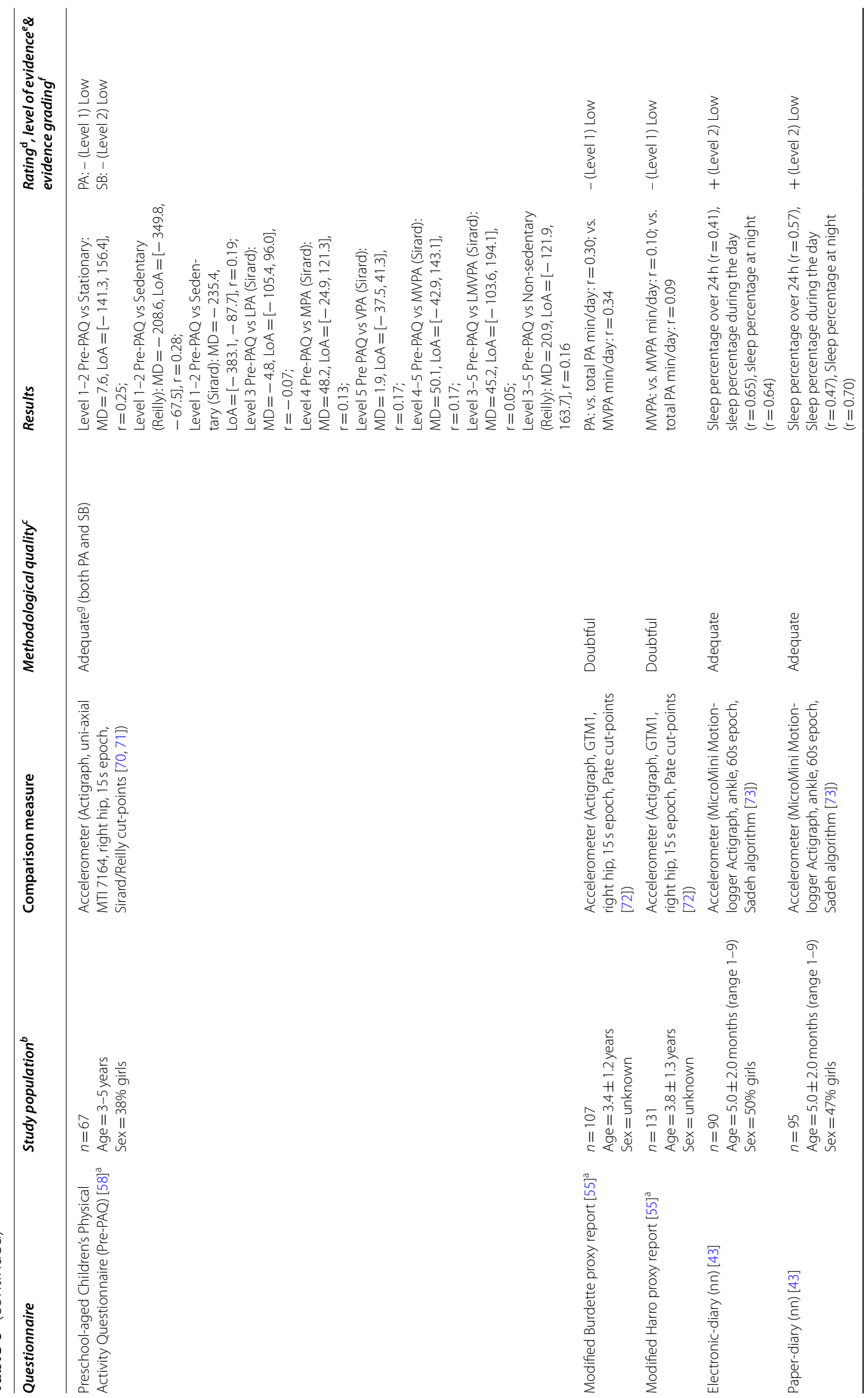




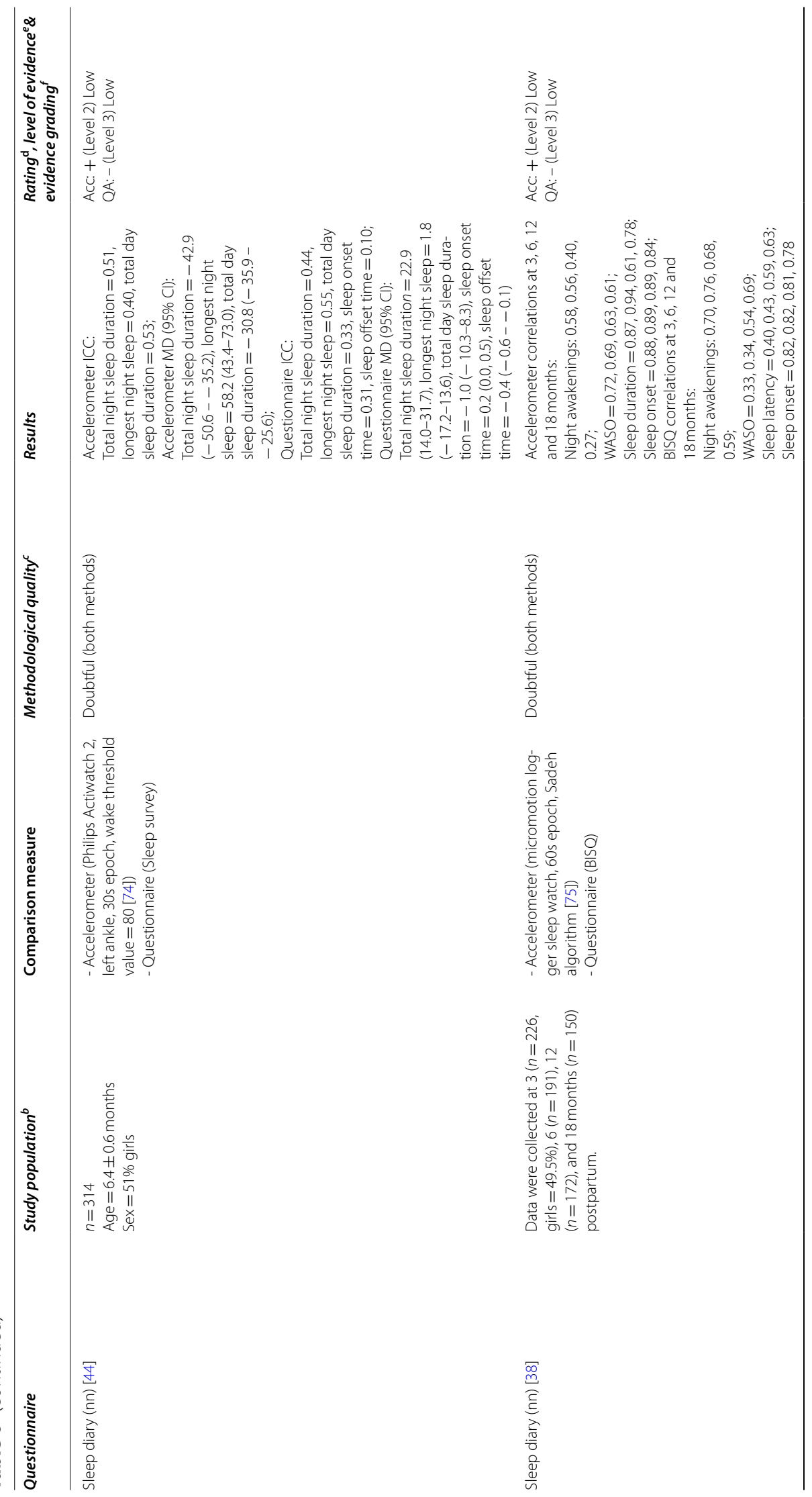




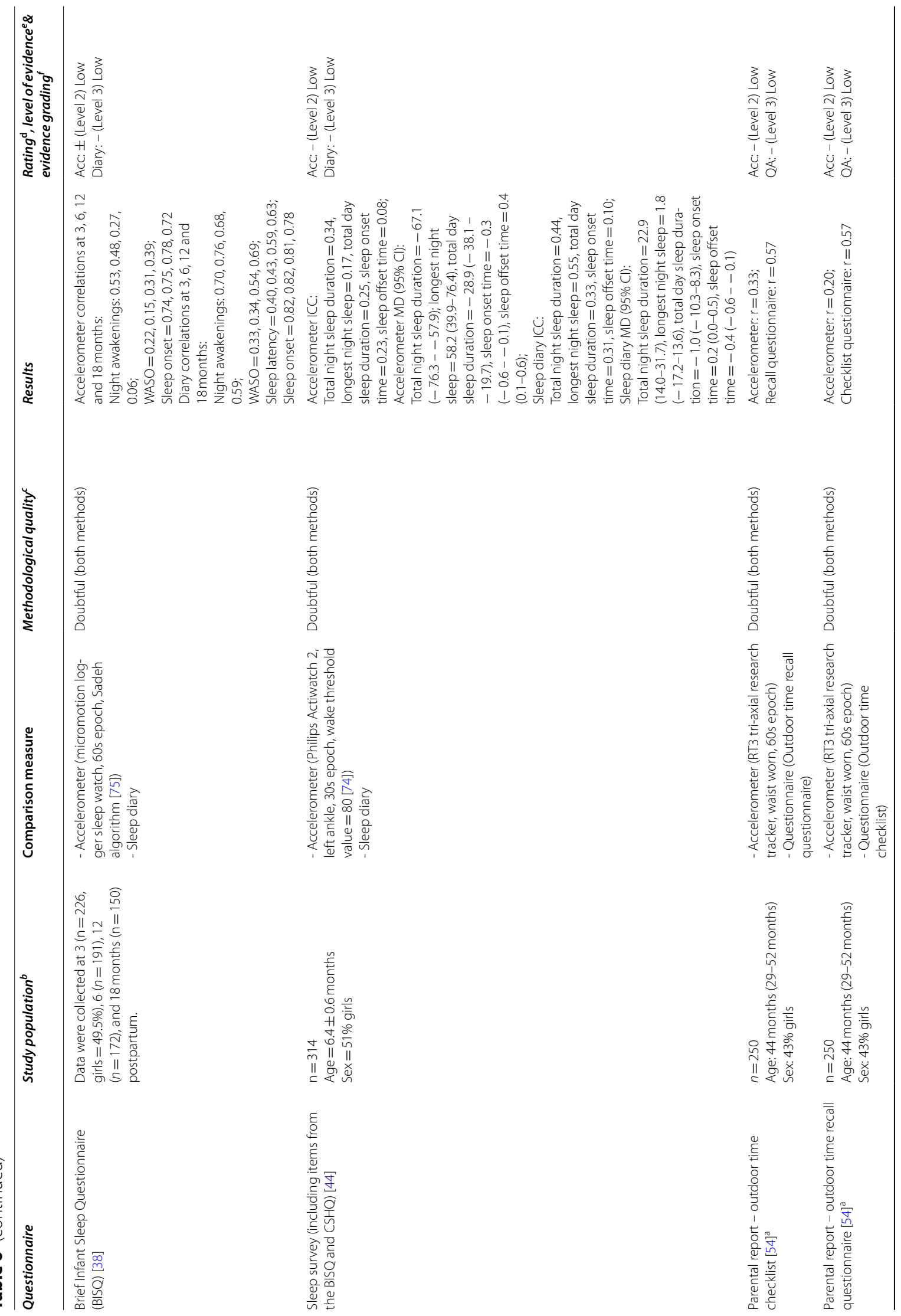




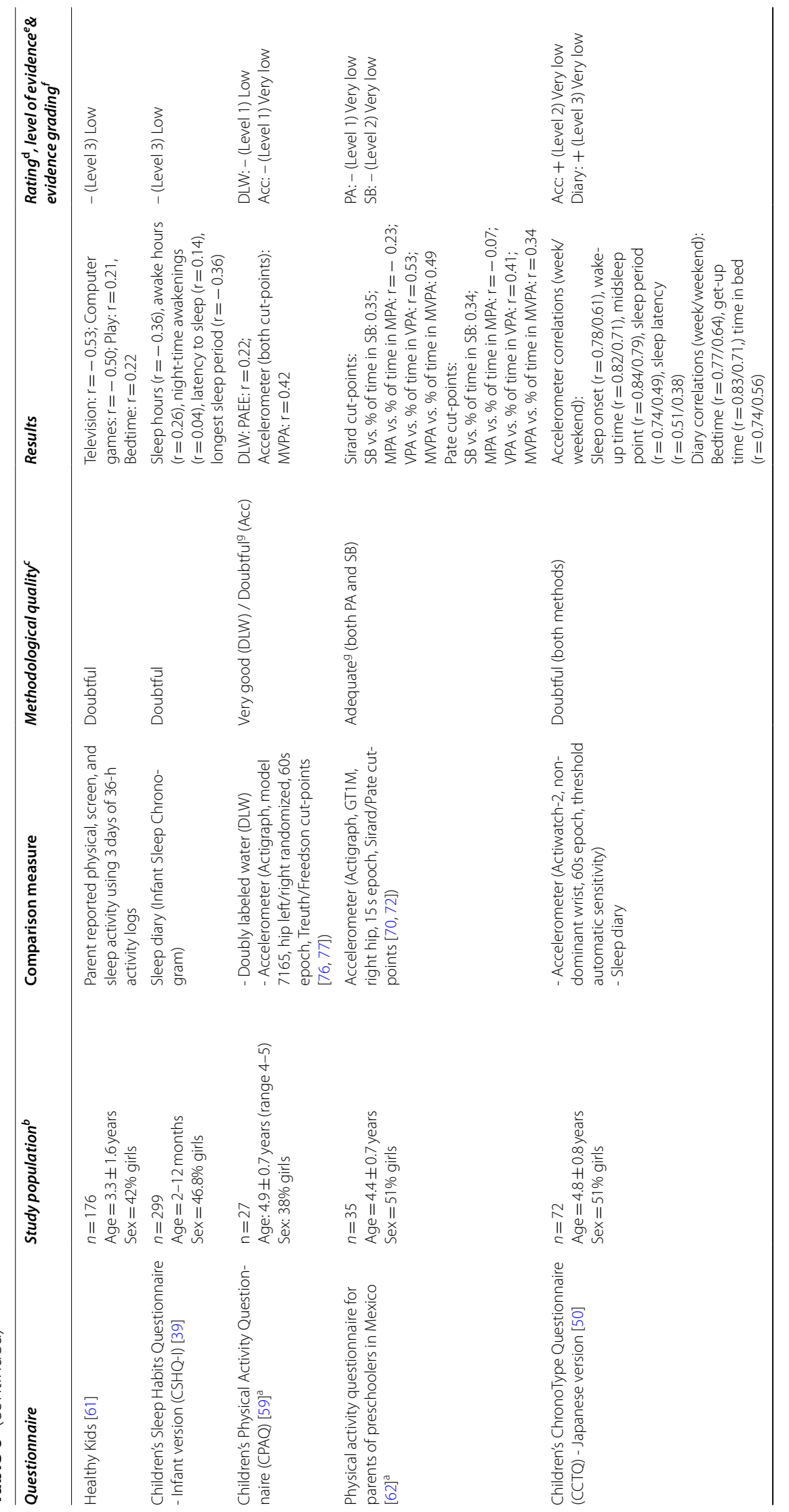




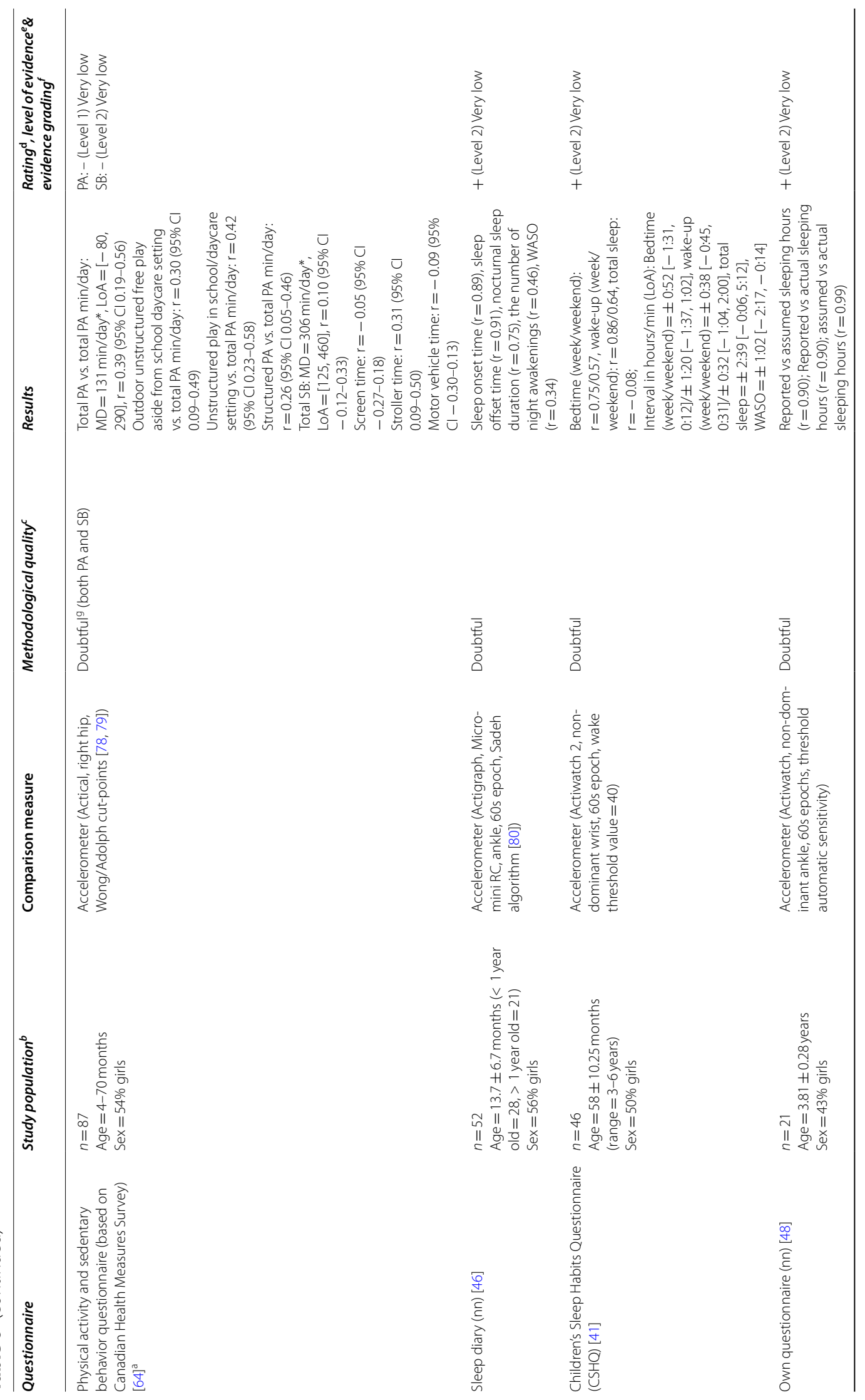




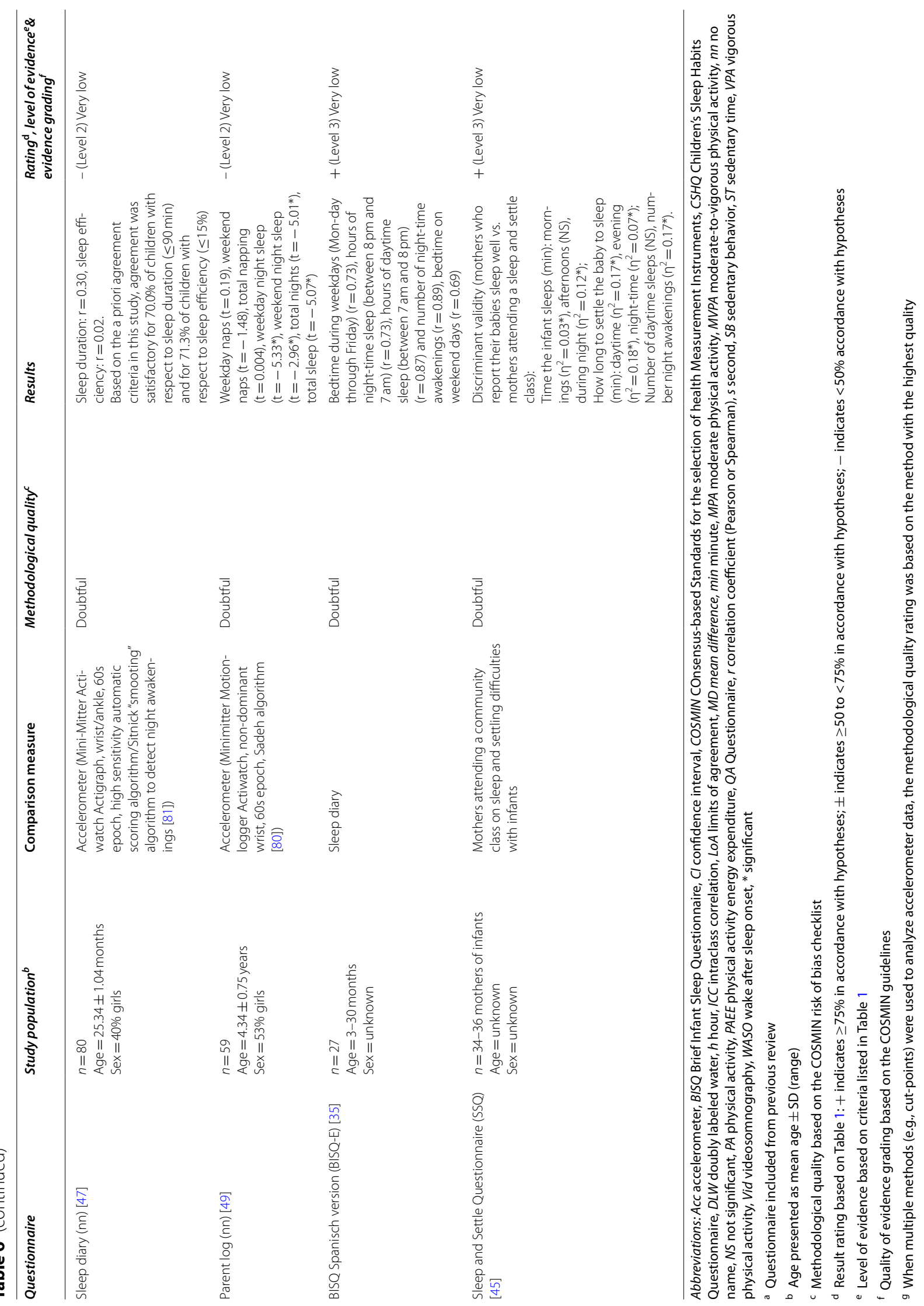


the content validity of the questionnaire $[36,51,52,60$, $65,66]$. Consequently, it remains unclear if the respondents (e.g., parents, youth health care providers) consider the content of the questionnaire as relevant, comprehensive and comprehensible [32]. Without evaluating content validity, there is no certainty that the questionnaire measures what it intends to measure $[26,31]$. It can be argued that it is difficult to proxy-report young children's sporadic and intermittent behaviors [17], and therefore it would be unrealistic to expect that questionnaires can be used to assess 24 -h movement behaviors very accurately in this target population. However, a more comprehensive development and evaluation of questionnaires (while including the target population and professionals in the process) would improve the quality of questionnaires. As appropriate measurement instruments are lacking, current evidence on young children's 24-h movement behaviors and the effects on their growth and development is limited [82]. Therefore, improving the quality of questionnaires and alternative (device-based) measures would strongly benefit research in this field, and thereby future public health recommendations.

Second, the quality of evidence was graded as low or very low for the majority of evaluated measurement properties of included questionnaires (i.e., 40 out of 49 evaluated measurement properties across all questionnaires), which makes it even more difficult to draw conclusions about the most appropriate available questionnaires. Although there are a number of questionnaires of which reliability (test-retest reliability: $n=4$, internal consistency: $n=1$ ) and/or validity (construct validity: $n=11$, structural validity: $n=2$ ) outcomes were considered sufficient, only three of these questionnaires received at least a moderate quality of evidence grading for one of the evaluated measurement properties. This concerns the internal consistency of the CSWS [30] and the structural validity of the CSHQ [40] and CSHQ-I [39]. Unfortunately, other measurement properties of these questionnaires received a low or very low quality of evidence grading (e.g., reliability), or were not included in our review since these were not assessed in 0- to 5-yearold children (e.g., construct validity and structural validity of the CSWS [51]). Hence, no definite conclusions about the appropriateness of these questionnaires to assess sleep behavior in young children can be drawn.

There are a few reasons that contributed to the low quality of evidence of questionnaires. First, quality of evidence was often downgraded because of the small sample sizes (<100 participants) included in studies (i.e., risk of imprecision) [30]. If multiple studies on the same measurement property of a questionnaire would have been available, pooling of study results would be possible, thereby solving this sample size issue [30].
Second, quality of evidence was often downgraded because of the doubtful methodology quality of studies (i.e., risk of bias) [30]. The lack of high-quality studies is consistent with findings from previous reviews by Hidding et al. [20, 21]. Common methodological limitations varied by evaluated measurement property. Concerning test-retest reliability, Pearson or Spearman correlations were often used without providing evidence that no systematic changes had occurred [30]. Furthermore, some reliability studies used inappropriate long time intervals between test and retest, e.g., 3 months, reducing the probability that children have remained stable in the interim period on the construct to be measured [30, 83]. The low methodological quality of construct validity studies was predominantly due to using comparator instruments with unknown or insufficient measurement properties. Most studies used, for example, non-validated diaries, or accelerometers without providing validated analyses methods (e.g., cut-points) in young children. Unfortunately, adequate comparator instruments with validated analyses methods are generally lacking in this young age group, making it difficult to ensure that comparator instruments measure the same constructs [13, 16, 29]. The doubtful methodological quality of content validity studies was predominantly due to the limited information reported on the methods used for the development of the questionnaire or the content validity evaluation. In particular, details on procedures of interviews or group meetings were lacking. For example, it was unclear whether interviewers were trained, whether data was coded independently, or whether data saturation was reached [31]. Moreover, not all aspects of content validity (i.e., relevance, comprehensiveness, and comprehensibility) were evaluated or the respondents (e.g., parents) were not included where appropriate.

It should be noted that some of the included questionnaires were not specifically aimed at assessing movement behaviors [53, 60, 61, 63, 65-67]. Consequently, frequency and/or duration of physical activity, sedentary behavior and/or sleep were only one of the many subconstructs in these questionnaires. The Healthy Kids $[60,61]$, for example, is intended to assess obesity risk. This questionnaire included items related to children's physical activity, sedentary behavior and sleep, as part of a larger questionnaire. Studies evaluating the content validity of such questionnaires considered the questionnaire as a whole, instead of each sub-construct separately $[60,65,66]$. Consequently, it was unclear whether all included items for physical activity, sedentary behavior and/or sleep were relevant and whether all key concepts were included (i.e., comprehensiveness). Future studies evaluating the content validity of questionnaires assessing multiple behaviors should evaluate each subscale or 
sub-construct separately to ensure an adequate evaluation of all included constructs [31].

\section{Strengths and limitations}

A strength of this review is the standardized quality assessment of included studies, using the COSMIN guidelines [28, 30, 31]. Another strength is that we included questionnaires for assessing all 24-h movement behaviors (i.e., physical activity, sedentary behavior and sleep). There is growing evidence that all 24-h movement behaviors should be targeted when aiming for optimal health [5-10]. The current review gives a clear overview of the available proxy-report questionnaires to monitor these behaviors in young children. Additionally, screening, data extraction and methodological quality assessment have each been done by at least two independent researchers, minimizing the chance of bias. However, our review also has some limitations. We only included studies published in English, disregarding studies published in other languages. Consequently, our review might not be representative for questionnaires available in nonEnglish speaking countries, although we only excluded a limited number of studies based on language. Furthermore, we rated the methodological quality of studies based on the information reported in each of the articles. When details on the methodology were lacking, studies received lower quality of evidence grades. We did not contact authors for additional information other than requesting the questionnaires that were used. Consequently, the quality of evidence of some studies might have been underestimated.

\section{Recommendations for future studies}

Given the lack of questionnaires for assessing movement behaviors in young children, we recommend future studies to develop proxy-report questionnaires targeted specifically at children aged $0-5$ years, including all 24-h movement behaviors. These questionnaires should preferably be tailored to fit the specific behavior of the subgroup (i.e., infants, toddlers or preschoolers), as movement behaviors quickly change during this period of rapid development.

When developing questionnaires, we strongly recommend future studies to evaluate the content validity of proxy-report questionnaires in a sample representing the target population (e.g., parents), including all components of content validity (i.e., relevance, comprehensiveness, and comprehensibility). We recommend these studies to use appropriate qualitative data collection methods, such as focus groups, interviews or concept mapping [31], and to evaluate the content validity of each subscale or sub-construct separately [31].

Next, high quality research on all other relevant measurement properties (both reliability and validity) of developed questionnaires is necessary. Future studies evaluating measurement properties of questionnaires are recommended to use standardized tools to increase study quality. We recommend the use of the COSMIN methodology for the design and reporting of these studies [84]. Specifically, high quality studies on measurement error, responsiveness, and cross-cultural validity of questionnaires are needed.

First, we recommend future studies that examine test-retest reliability to additionally report the measurement error, as both measurement properties can be calculated based on the same data and study design [84]. These studies should use at least two independent measurements with similar test conditions and an appropriate time interval, according to previous recommendations for youth physical activity questionnaires: $>1$ day and $<1$ week for questionnaires recalling the previous day, $>1$ day and $<2$ weeks for questionnaires recalling the recalling the previous week [19]. The preferred statistical method to assess the measurement error of continuous scores is the calculation of the standard error of measurement (SEM) [30]. For categorical scores we recommend calculating the percentage positive and negative agreement [84].

Second, more studies examining responsiveness of questionnaires are needed, reflecting longitudinal validity. Similar to construct validity, it is important to define hypotheses in advance when assessing responsiveness of a questionnaire, to enable drawing unbiased conclusions [84].

Third, we recommend future studies evaluating translated or culturally adapted questionnaires to examine cross-cultural validity. These studies are recommended to provide a clear description of the characteristics that should be similar in the different subgroups (e.g., demographics such as age) and provide clear information on the performance of the analysis (e.g. software program used, criteria for model fit) [84].

Last, as current studies evaluating the criterion and construct validity of questionnaires are limited due to a lack of comparators with sufficient measurement properties, we recommend future studies to improve comparator instruments and analysis methods to assess 24-h movement behaviors (e.g., accelerometer data analyses). Subsequently, improving the quality of questionnaires and alternative measurement methods would strongly benefit research in this field. 


\section{Conclusion}

None of the 37 proxy-report questionnaires included in this review were considered valid and/or reliable for assessing one or more movement behaviors in children aged $0-5$ years. The lack of high-quality methodological studies that evaluate all relevant measurement properties of developed questionnaires hampers our ability to draw definite conclusions about the best available questionnaires. In addition, questionnaires for assessing 24-h movement behaviors in 0- to 5-year-olds are scarce. Thus, high-quality studies are required aimed to develop proxy-report questionnaires for this age group and to evaluate their measurement properties, starting with content validity. When sufficient content validity is established, the remaining relevant measurement properties (i.e., reliability, validity, responsiveness) should be evaluated [31, 84]. Until valid and reliable proxy-report questionnaires are available, caution is needed when interpreting results of research using proxy-reported physical activity, sedentary behavior and sleep in this young age group.

\section{Supplementary Information}

The online version contains supplementary material available at https://doi. org/10.1186/s12966-022-01251-x.

\section{Additional file 1.}

Additional file 2

Acknowledgements

Not applicable.

\section{Authors' contributions}

$J A, T A, J G, A V$ and $M C$ conceived the study. JA and TA screened all articles for eligibility. JA, JG and AV extracted the data. JA, TA, MC and AL rated the methodological quality of studies. JA completed data analysis. JA drafted the manuscript. TA, JG, AV and MC revised and edited significant sections of the manuscript. All authors reviewed and approved the final manuscript.

\section{Funding}

This review is part of the 'My Little Moves' project that is funded by ZonMw (546003008) and the Bernard van Leer foundation. The funding bodies had no role in the design of the study; in the collection, analysis, and interpretation of data; or in the writing of the manuscript.

\section{Availability of data and materials}

The data that support the findings of this review are available from the corresponding author upon reasonable request.

\section{Declarations}

Ethics approval and consent to participate

Not applicable.

\section{Consent for publication}

Not applicable.

\section{Competing interests}

The authors declare that they have no competing interests.

\section{Author details}

${ }^{1}$ Department of Public and Occupational Health, Amsterdam UMC, Vrije Universiteit Amsterdam, Amsterdam Public Health research institute, Van der Boechorststraat 7, 1081, BT, Amsterdam, The Netherlands. ${ }^{2}$ Department of Health Promotion, NUTRIM School of Nutrition and Translational Research in Metabolism, Maastricht University, PO Box 616, 6200, MD, Maastricht, The Netherlands. ${ }^{3}$ Public Health Service Amsterdam, Sarphati Amsterdam, 1018, WT, Amsterdam, The Netherlands. ${ }^{4}$ Department of Sociology, University of Amsterdam, 1018, WV, Amsterdam, The Netherlands.

Received: 28 July 2021 Accepted: 1 February 2022

Published online: 14 February 2022

\section{References}

1. Hills AP, King NA, Armstrong TP. The contribution of physical activity and sedentary Behaviours to the growth and development of children and adolescents. Sports Med. 2007;37(6):533-45.

2. Jones RA, et al. Tracking physical activity and sedentary behavior in childhood: a systematic review. Am J Prev Med. 2013;44(6):651-8.

3. Timmons BW, et al. Systematic review of physical activity and health in the early years (aged 0-4 years). Appl Physiol Nutr Metab. 2012;37(4):773-92.

4. Chaput JP, et al. Systematic review of the relationships between sleep duration and health indicators in the early years (0-4 years). BMC Public Health. 2017;17(Suppl 5):855.

5. Chaput JP, et al. Importance of all movement behaviors in a 24 hour period for overall health. Int J Environ Res Public Health. 2014;11(12):12575-81.

6. Kuzik N, et al. Systematic review of the relationships between combinations of movement behaviours and health indicators in the early years (0-4 years). BMC Public Health. 2017;17(5):849.

7. Tremblay MS, et al. Canadian 24-hour movement guidelines for children and youth: an integration of physical activity, sedentary behaviour, and sleep. Appl Physiol Nutr Metab. 2016;41 (6 Suppl 3):S311-27.

8. Tremblay MS, et al. Canadian 24-hour movement guidelines for the early years (0-4 years): an integration of physical activity, sedentary behaviour, and sleep. BMC Public Health. 2017;17(Suppl 5):874.

9. World Health Organization. Guidelines on physical activity, sedentary behaviour and sleep for children under 5 years of age. Geneva: World Health Organization; 2019. https://apps.who.int/iris/handle/10665/ 311664. License: CC BY-NC-SA 3.0 IGO.

10. Rollo S, Antsygina O, Tremblay MS. The whole day matters: understanding 24-hour movement guideline adherence and relationships with health indicators across the lifespan. J Sport Health Sci. 2020;9(6):493-510.

11. Sirard JR, Pate RR. Physical activity assessment in children and adolescents. Sports Med. 2001;31(6):439-54.

12. Sadeh A, lii. Sleep assessment methods. Monogr Soc Res Child Dev. 2015;80(1):33-48.

13. Bruijns BA, et al. Infants' and toddlers' physical activity and sedentary time as measured by accelerometry: a systematic review and meta-analysis. Int J Behav Nutr Phys Act. 2020;17(1):14.

14. Trost SG. State of the art reviews: measurement of physical activity in children and adolescents. Am J Lifestyle Med. 2007;1 (4):299-314.

15. Trost SG, Mclver KL, Pate RR. Conducting accelerometer-based activity assessments in field-based research. Med Sci Sports Exerc. 2005;37(11 Suppl):S531-43.

16. Cliff DP, Reilly JJ, Okely AD. Methodological considerations in using accelerometers to assess habitual physical activity in children aged $0-5$ years. $J$ Sci Med Sport. 2009;12(5):557-67.

17. Kohl HW, Fulton JE, Caspersen CJ. Assessment of physical activity among children and adolescents: a review and synthesis. Prev Med. 2000;31(2):S54-76.

18. Nascimento-Ferreira MV, et al. Validity and reliability of sleep time questionnaires in children and adolescents: a systematic review and meta-analysis. Sleep Med Rev. 2016;30:85-96.

19. Chinapaw MJ, et al. Physical activity questionnaires for youth: a systematic review of measurement properties. Sports Med. 2010;40(7):539-63. 
20. Hidding LM, et al. Systematic review of childhood sedentary behavior questionnaires: what do we know and what is next? Sports Med 2017:47(4):677-99.

21. Hidding LM, et al. An updated systematic review of childhood physical activity questionnaires. Sports Med. 2018:48(12):2797-842.

22. Spruyt K, Gozal D. Pediatric sleep questionnaires as diagnostic or epidemiological tools: a review of currently available instruments. Sleep Med Rev. 2011;15(1):19-32.

23. Sen T, Spruyt K. Pediatric sleep tools: an updated literature review. Front Psychiatry. 2020;11:317.

24. Byrne R, Terranova CO, Trost SG. Measurement of screen time among young children aged 0-6 years: a systematic review. Obes Rev. 2021;22(8):e13260. https://doi.org/10.1111/obr.13260.

25. Page MJ, Moher D, Bossuyt PM, Boutron I, Hoffmann TC, Mulrow CD, et al. PRISMA 2020 explanation and elaboration: updated guidance and exemplars for reporting systematic reviews. BMJ. 2021;372:1-36. https:// doi.org/10.1136/bmj.n160.

26. De Vet $\mathrm{H}$, et al. Measurement in medicine: A practical guide. Measurement in Medicine: A Practical Guide; 2011. p. 1-338.

27. Edwards JR, Bagozzi RP. On the nature and direction of relationships between constructs and measures. Psychol Methods. 2000;5(2):155.

28. Mokkink LB, et al. COSMIN risk of Bias checklist for systematic reviews of patient-reported outcome measures. Qual Life Res. 2018;27(5):1171-9.

29. Lettink, A., et al., Systematic review of accelerometer-based methods for 24-hour physical behavior assessment in young children (0-5-years-old). Article in preparation.

30. Prinsen CAC, et al. COSMIN guideline for systematic reviews of patientreported outcome measures. Qual Life Res. 2018;27(5):1147-57.

31. Terwee $C B$, et al. COSMIN methodology for evaluating the content validity of patient-reported outcome measures: a Delphi study. Qual Life Res. 2018;27(5):1159-70.

32. Mokkink LB, et al. The COSMIN study reached international consensus on taxonomy, terminology, and definitions of measurement properties for health-related patient-reported outcomes. J Clin Epidemiol. 2010;63(7):737-45.

33. Field A. Discovering statistics using IBM SPSS statistics. London: SAGE Publications; 2013. ISBN: 9781446249185 . https://books.google.nl/books? $h|=n| \&|r=\& i d=c 0 W k 9| u B m A o C \& o i=f n d \& p g=P P 2 \& d q=$ reference + Field+A.+Discovering + statistics+using+IBM+SPSS+statistics:+sage\% $3 \mathrm{~B}+2013 . \& \mathrm{ots}=\mathrm{LcD} \mid \mathrm{JO} 2 \mathrm{w} \_\mathrm{F} \& \mathrm{sig}=\mathrm{UzP}$ USLsTGV9BQuM42HaOfwcnfc $\&$ redir_esc=y $\# v=$ onepage $\& \mathrm{q}=$ reference $\% 20$ Field $\% 20 \mathrm{~A} . \% 20$ Discovering $\%$ 20statistics\%20using\%20IBM\%20SPSS\%20statistics\%3A\%20sage\%3B\% 202013.\&f=false.

34. Field AP. Discovering statistics using SPSS : (and sex and drugs and rock ' $n$ ' roll). 3rd ed. ed. Introducing statistical methods. Los Angeles [i.e. Thousand Oaks, Calif.]: SAGE Publications; 2009.

35. Cassanello P, et al. Adaptation and study of the measurement properties of a sleep questionnaire for infants and pre-school children. Anales de Pediatria. 2018;89(4):230-7.

36. Dhakal AK, et al. A Nepali translation of brief infant sleep questionnaire (BISQ) for assessment of sleep in infants and toddlers: a preliminary report. J Kathmandu Med Coll. 2014;3(3):102-6.

37. Boran, P., et al., Translation into Turkish of the expanded version of the "brief infant sleep questionnaire" and its application to infants. 2014.

38. Tikotzky L, Volkovich E. Infant nocturnal wakefulness: a longitudinal study comparing three sleep assessment methods. Sleep. 2019;42(1):zsy191.

39. Dias CC, Figueiredo B, Pinto TM. Children's sleep habits questionnaire infant version. J Pediatr. 2018;94(2):146-54

40. Liu Z, et al. Reliability and validity of the children's sleep habits questionnaire in preschool-aged Chinese children. Sleep Biol Rhythms. 2014;12(3):187-93.

41. Perpétuo C, Fernandes M, Veríssimo M. Comparison between actigraphy records and parental reports of Child's sleep. Front Pediatr. 2020:8(567390):1-8. https://doi.org/10.3389/fped.2020.567390.

42. Camerota M, Tully KP, Grimes M, Gueron-Sela N, Propper CB. Assessment of infant sleep: how well do multiple methods compare? Sleep. 2018;41(10):1-12. https://doi.org/10.1093/sleep/zsy146.

43. Muller $\mathrm{S}$, et al. Parental report of infant sleep behavior by electronic versus paper-and-pencil diaries, and their relationship to actigraphic sleep measurement. J Sleep Res. 2011;20(4):598-605.
44. Quante M, Hong B, von Ash T, Yu X, Kaplan ER, Rueschman M, Jackson CL, Haneuse S, Davison K, Taveras EM, Redline S. Associations between parentreported and objectively measured sleep duration and timing in infants at age 6 months. Sleep. 2021;44(4):1-10. https://doi.org/10.1093/ sleep/zsaa217.

45. Matthey S. The sleep and settle questionnaire for parents of infants: psychometric properties. J Paediatr Child Health. 2001:37(5):470-5.

46. Asaka Y, Takada S. Comparing sleep measures of infants derived from parental reports in sleep diaries and acceleration sensors. Acta Paediatr. 2011;100(8):1158-63.

47. Bélanger $M-\dot{E}$, et al. Investigating the convergence between actigraphy, maternal sleep diaries, and the child behavior checklist as measures of sleep in toddlers. Front psychiatry. 2014;5:158.

48. Sekine $M$, et al. The validity of sleeping hours of healthy young children as reported by their parents. J Epidemiol. 2002;12(3):237-42.

49. Lam JC, et al. Defining the roles of actigraphy and parent logs for assessing sleep variables in preschool children. Behav Sleep Med. 2011;9(3):184-93.

50. Ishihara K, Doi Y, Uchiyama M. The reliability and validity of the Japanese version of the Children's ChronoType questionnaire (CCTQ) in preschool children. Chronobiol Int. 2014;31(9):947-53.

51. LeBourgeois MK, Harsh JR. Development and psychometric evaluation of the Children's sleep-wake scale. Sleep Health. 2016;2(3):198-204.

52. Howie EK, McNally S, Straker LM. Exploring the reliability and validity of the TechU-Q to evaluate device and purpose specific screen use in preschool children and parents. J Child Fam Stud. 2020;29(10):2879-89.

53. Carson V, et al. Psychometric properties of a parental questionnaire for assessing correlates of toddlers' physical activity and sedentary behavior. Meas Phys Educ Exerc Sci. 2017;21(4):190-200.

54. Burdette HL, Whitaker RC, Daniels SR. Parental report of outdoor playtime as a measure of physical activity in preschool-aged children. Arch Pediatr Adolesc Med. 2004;158(4):353-7.

55. Rice KR, Joschtel B, Trost SG. Validity of family child care providers' proxy reports on children's physical activity. Child Obes. 2013;9(5):393-8.

56. Bonn SE, et al. Feasibility of a novel web-based physical activity questionnaire for young children. Pediatr Rep. 2012;4(4):e37-7.

57. Bingham DD, et al. Reliability and validity of the early years physical activity questionnaire (EY-PAQ). Sports. 2016;4(2):30.

58. Dwyer GM, et al. The validity and reliability of a home environment preschool-age physical activity questionnaire (pre-PAQ). Int J Behav Nutr Phys Act. 2011;8(1):86.

59. Corder $\mathrm{K}$, et al. Is it possible to assess free-living physical activity and energy expenditure in young people by self-report? Am J Clin Nutr. 2009;89(3):862-70.

60. Townsend, M.S., et al., Obesity risk for young children: development and initial validation of an assessment tool for participants of federal nutrition programs. 2014.

61. Townsend MS, et al. An obesity risk assessment tool for young children: validity with BMI and nutrient values. J Nutr Educ Behav. 2018;50(7):705-17.

62. Bacardi-Gascón M, et al. Assessing the validity of a physical activity questionnaire developed for parents of preschool children in Mexico. J Health Popul Nutr. 2012;30(4):439.

63. González-Gil E, et al. Reliability of primary caregivers reports on lifestyle behaviours of E uropean pre-school children: the T oy B ox-study. Obes Rev. 2014;15:61-6.

64. Sarker $\mathrm{H}$, et al. Validation of parent-reported physical activity and sedentary time by accelerometry in young children. BMC Res Nnotes. 2015:8(1):735.

65. Chia M, Tay LY, Chua TBK. The development of an online surveillance of digital media use in early childhood questionnaire-SMALLQ ${ }^{\text {TM }}$-for Singapore; 2019.

66. Goncalves W, et al. Cross-cultural adaptation of instruments measuring Children's movement behaviors and parenting practices in Brazilian families. Int J Environ Res Public Health. 2021;18(1):239.

67. Hinkley T, et al. The HAPPY study: development and reliability of a parent survey to assess correlates of preschool children's physical activity. J Sci Med Sport. 2012;15(5):407-17.

68. Costa $\mathrm{S}$, et al. Calibration and validation of the ActiGraph GT3X+ in 2-3 year olds. J Sci Med Sport. 2014;17(6):617-22. 
69. Pate RR, et al. Validation and calibration of an accelerometer in preschool children. Obesity (Silver Spring). 2006;14(11):2000-6.

70. Sirard JR, et al. Calibration and evaluation of an objective measure of physical activity in preschool children. J Phys Act Health. 2005;2(3):345-57.

71. Reilly JJ, et al. An objective method for measurement of sedentary behavior in 3- to 4-year olds. Obes Res. 2003;11(10):1155-8.

72. Pate RR, O'Neill JR, Mitchell J. Measurement of physical activity in preschool children. Med Sci Sports Exerc. 2010;42(3):508-12.

73. Sadeh A, et al. Actigraphically based automatic bedtime sleep-wake scoring: validity and clinical applications. J Ambul Monit. 1989;2(3):209-16.

74. So K, et al. Actigraphy correctly predicts sleep behavior in infants who are younger than six months, when compared with polysomnography. Pediatr Res. 2005;58(4):761-5.

75. Sadeh A, et al. Activity-based assessment of sleep-wake patterns during the 1st year of life. Infant Behav Dev. 1995;18(3):329-37.

76. Treuth MS, et al. Defining accelerometer thresholds for activity intensities in adolescent girls. Med Sci Sports Exerc. 2004;36(7):1259-66.

77. Freedson PS, Melanson E, Sirard J. Calibration of the computer science and applications. Inc accelerometer Med Sci Sports Exerc. 1998;30(5):777-81.

78. Wong SL, et al. Actical accelerometer sedentary activity thresholds for adults. J Phys Act Health. 2011;8(4):587-91.

79. Adolph AL, et al. Validation of uniaxial and triaxial accelerometers for the assessment of physical activity in preschool children. J Phys Act Health. 2012;9(7):944-53.

80. Sadeh A, Sharkey M, Carskadon MA. Activity-based sleep-wake identification: an empirical test of methodological issues. Sleep. 1994;17(3):201-7.

81. Sitnick SL, Goodlin-Jones BL, Anders TF. The use of actigraphy to study sleep disorders in preschoolers: some concerns about detection of nighttime awakenings. Sleep. 2008;31(3):395.

82. Veldman SL, Paw MJCA, Altenburg TM. Physical activity and prospective associations with indicators of health and development in children aged $<5$ years: a systematic review. Int J Behav Nutr Phys Act. 2021;18(1):1-11.

83. Streiner DL, Norman GR, Cairney J. Health measurement scales: a practical guide to their development and use. USA: Oxford University Press; 2015

84. Mokkink, L.B., et al., COSMIN study design checklist for patient-reported outcome measurement instruments. Department of Epidemiology and Biostatistics, 2019

\section{Publisher's Note}

Springer Nature remains neutral with regard to jurisdictional claims in published maps and institutional affiliations.

Ready to submit your research? Choose BMC and benefit from:

- fast, convenient online submission

- thorough peer review by experienced researchers in your field

- rapid publication on acceptance

- support for research data, including large and complex data types

- gold Open Access which fosters wider collaboration and increased citations

- maximum visibility for your research: over 100M website views per year

At BMC, research is always in progress.

Learn more biomedcentral.com/submissions 